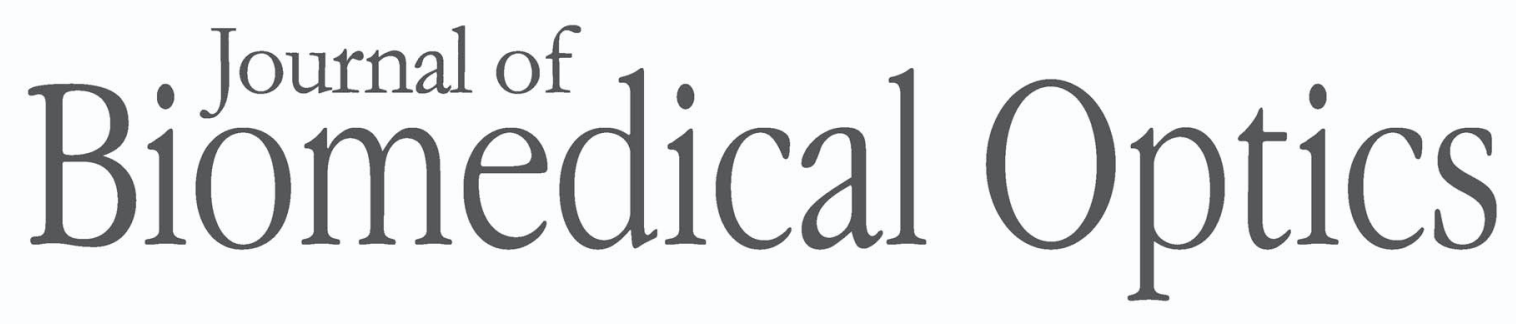

SPIEDigitalLibrary.org/jbo

\title{
Tissue polarimetry: concepts, challenges, applications, and outlook
}

Nirmalya Ghosh

I. Alex Vitkin 


\title{
Tissue polarimetry: concepts, challenges, applications, and outlook
}

\author{
Nirmalya Ghosh ${ }^{\mathrm{a}}$ and I. Alex Vitkin ${ }^{\mathrm{b}, \mathrm{c}, \mathrm{d}}$ \\ a Indian Institute of Science Education and Research (IISER), Department of Physical Sciences, Kolkata, Mohanpur, \\ West Bengal, India \\ ${ }^{b}$ Division of Biophysics and Bioimaging, Ontario Cancer Institute, Princess Margaret Hospital, 7th Floor Rm. 415, \\ 610 University Ave, Toronto, Ontario, Canada M5G 2M9 \\ 'Department of Medical Biophysics, University of Toronto, 610 University Avenue, Toronto, Ontario M5G 2M9 Canada \\ ${ }^{\mathrm{d}}$ Department of Radiation Oncology, University of Toronto, 610 University Avenue, Toronto, \\ Ontario M5G 2M9, Canada
}

\begin{abstract}
Polarimetry has a long and successful history in various forms of clear media. Driven by their biomedical potential, the use of the polarimetric approaches for biological tissue assessment has also recently received considerable attention. Specifically, polarization can be used as an effective tool to discriminate against multiply scattered light (acting as a gating mechanism) in order to enhance contrast and to improve tissue imaging resolution. Moreover, the intrinsic tissue polarimetry characteristics contain a wealth of morphological and functional information of potential biomedical importance. However, in a complex random medium-like tissue, numerous complexities due to multiple scattering and simultaneous occurrences of many scattering and polarization events present formidable challenges both in terms of accurate measurements and in terms of analysis of the tissue polarimetry signal. In order to realize the potential of the polarimetric approaches for tissue imaging and characterization/diagnosis, a number of researchers are thus pursuing innovative solutions to these challenges. In this review paper, we summarize these and other issues pertinent to the polarized light methodologies in tissues. Specifically, we discuss polarized light basics, Stokes-Muller formalism, methods of polarization measurements, polarized light modeling in turbid media, applications to tissue imaging, inverse analysis for polarimetric results quantification, applications to quantitative tissue assessment, etc. $\Theta 2011$ Society of Photo-Optical Instrumentation Engineers (SPIE). [DOI: 10.1117/1.3652896]
\end{abstract}

Keywords: polarization; multiple scattering; turbid polarimetry; light transport; Monte Carlo simulations; Stokes vector, Mueller matrix, polar decomposition; biological and medical applications.

Paper 11272TR received May 30, 2011; revised manuscript received Aug. 24, 2011; accepted for publication Sep. 22, 2011; published online Nov. 14, 2011.

\section{Introduction}

Polarized light has played important roles in our understanding of the nature of electromagnetic waves, ${ }^{1}$ elucidating the threedimensional characteristics of chemical bonds ${ }^{2}$ uncovering the asymmetric (chiral) nature of biological molecules, ${ }^{3}$ determining sugar concentrations in industrial processes,${ }^{4}$ quantifying protein properties in solutions, ${ }^{5}$ supplying a variety of nondestructive evaluation methods, ${ }^{6}$ developing advanced concepts such as polarization entropy, ${ }^{7}$ contributing to remote sensing in meteorology and astronomy, ${ }^{8,9}$ and differentiating between normal and precancerous cells in superficial tissue layers, ${ }^{10}$ as well as other biomedical applications. ${ }^{11,12}$ Traditional polarimetry is well suited for applications in clear media and for studies of surfaces; however, multiple scattering in optically thick turbid media such as biological tissues causes extensive depolarization that confounds the established techniques. Further, even if some residual polarization signal can be measured, ${ }^{13}$ multiple scattering also alters the polarization state; for example, by scattering-induced diattenuation and by scatteringinduced changes in the orientation of the linear polarization vector which appears as optical rotation. ${ }^{14}$ The presence of other

Address all correspondence to: Nirmalya Ghosh, IISER Kolkata, Mohanpur Campus, Department of Physical Sciences, PO BCKV, Kolkata, West Bengal 741252, India; Tel: 91-9734678247; Fax: 91332587 3020; E-mail: nghosh@iiserkol.ac.in. simultaneously-occurring polarization effects further compromise quantitative polarimetry; in tissues, these include linear birefringence due to anisotropic muscle fibers and structural proteins, and optical rotation due to optically active (chiral) molecules and structures. ${ }^{15,16}$ Thus, although a wealth of interesting tissue properties can potentially be probed with polarized light, accurate measurements and data analysis leading to unique interpretation of the polarization parameters are difficult, hindering the utility of polarimetric bulk tissue characterization studies.

Some inroads in biomedical polarimetry have been made in the context of optical imaging, specifically using polarization gating to separate out and potentially remove the multiply scattered (depolarized) component of the light beam in order to enhance contrast and to improve tissue imaging resolution. ${ }^{12}$ As discussed below, this has proven moderately successful in selected applications, provided that proper attention is paid to the optimal choice of incident polarization states (e.g., linear versus circular), polarization detection schemes (e.g., Stokes versus Mueller polarimetry), geometry of detection (e.g., transmission versus reflection), etc. For example, some promising results have been reported in skin imaging, whereby a dermatologist uses polarization imaging to selectively concentrate on either surface irregularities or alternatively on deeper epidermal/dermal

1083-3668/2011/16(11)/110801/29/\$25.00 (C) 2011 SPIE 
layers. ${ }^{17}$ Further, polarization effects are extensively used in various forms of biomedical light microscopy, where one is dealing with thin fixed tissue slices, thus obviating the complicating effects of multiple scattering in bulk tissues by physical sectioning. But overall, the full potential of polarization imaging in biomedicine has not been realized, for reasons similar to the polarimetric tissue characterization studies mentioned above. To summarize, these include: 1. extensive loss of polarization signal engendered by tissue multiple scattering, 2 . complicated nature of polarization effects in tissue, including simultaneous multiple effects, 3. difficulties in measuring typically small tissue polarization signals, 4. challenges in analysis and quantification of measured signals or images, 5. complexities in understanding and interpreting tissue polarimetry results, and 6. scarcity of data on detailed polarimetric properties of various tissues and their effects on polarized light propagation.

Driven by polarimetry's biomedical potential, a number of researchers are pursuing innovative solutions to these challenges. In this review paper, we summarize these and other issues pertinent to the polarized light methodologies in tissues. Specifically, we discuss polarized light basics, Stokes-Muller formalism, methods of polarization measurements, polarized light modeling in turbid media, applications to tissue imaging, inverse matrix analysis for polarimetric results quantification and applications to quantitative tissue assessment, etc. The intent of the paper is to explain the basics of polarimetry, summarize its current state of research, provide selected illustrative examples, facilitate insight and understanding of the observed trends and findings, indicate the inconsistencies and outstanding issues, and point toward the future of the field. Biomedical polarimetry is still at a relatively early stage of development, with much of its promising potential currently unrealized; it is hoped that this paper will stimulate new ideas and encourage further research into this promising field.

\section{Polarization Basics and the Different Polarimetry Formalisms}

Definitions of polarized light and its properties are described in voluminous literature. ${ }^{6,7,18,19}$ Briefly, polarization is a property that arises out of the transverse (and vector) nature of the electromagnetic radiation and it describes the shape and the orientation of the locus of the electric field vector $(\vec{E})$ extremity as a function of time, at a given point of space. The classical concept of polarized light thus represents the state of polarization of a light wave as a function of the temporal evolution of its electric field vector $\overrightarrow{\mathrm{E}}$. If the vector extremity describes a stationary curve during the observation or measurement time, the wave is called polarized. It is called unpolarized if the extremity of vector $\overrightarrow{\mathrm{E}}$ exhibits random positions. Note that in the corresponding quantum mechanical description, instead of the field vector, polarization is described for individual photons (energy quanta). It is assumed that individual photons are completely polarized, and accordingly their polarization is described by a state vector. ${ }^{1,6}$ The superposition of many such photon states yield the resulting polarization observed for the classical wave. Thus the quantum mechanical view of polarization and the corresponding classical formalisms are mutually consistent.

Mathematical formalisms (in the classical approach) dealing with propagation of polarized light and its interaction with any optical system can be described by two formalisms; the Jones calculus $^{1,6,18}$ which is a field-based representation (assumes coherent addition of the amplitudes and phases of the waves) and the Stokes-Mueller calculus ${ }^{1,6,18-21}$ that is an intensity-based representation (assumes an incoherent addition of wave intensities). A major drawback of the Jones formalism is that it deals with pure polarization states only and cannot handle partial polarizations and thus depolarizing interactions (which are common in biological tissues). Thus its use in tissue polarimetry is limited. The general case, which does include polarization loss, can be better addressed by the Stokes-Mueller formalism as described below.

\subsection{Stokes Mueller Formalism}

In this formalism, the polarization state of the light beam is represented by four measurable quantities (intensities) grouped in a $4 \times 1$ vector, known as the Stokes vector ${ }^{6,18-21}$ (introduced by Stokes in 1852). The four Stokes parameters are defined relative to the following six intensity measurements $(I)$ performed with ideal polarizers: $I_{H}$, horizontal linear polarizer ( $\left.0 \mathrm{deg}\right) ; I_{V}$, vertical linear polarizer (90 deg); $I_{P}, 45 \mathrm{deg}$ linear polarizer; $I_{M}$, $135 \mathrm{deg}$ ( $-45 \mathrm{deg}$ ) linear polarizer; $I_{R}$, right circular polarizer, and $I_{L}$, left circular polarizer. The Stokes vector $(\mathbf{S})$ is defined as $^{18-21}$

$$
\mathbf{S}=\left[\begin{array}{c}
I \\
Q \\
U \\
V
\end{array}\right]=\left[\begin{array}{c}
I_{H}+I_{V} \\
I_{H}-I_{V} \\
I_{P}-I_{M} \\
I_{R}-I_{L}
\end{array}\right],
$$

where $I, Q, U$, and $V$ are Stokes vector elements. $I$ is the total detected light intensity which corresponds to addition of the two orthogonal component intensities, $Q$ is the difference in intensity between horizontal and vertical polarization states, $U$ is the portion of the intensity that corresponds to the difference between intensities of linear $+45 \mathrm{deg}$ and $-45 \mathrm{deg}$ polarization states, and $V$ is the difference between intensities of right circular and left circular polarization states.

In the Stokes formalism, the following polarization parameters of any light beam are defined: ${ }^{6,18-21}$

Net degree of polarization

$$
\mathrm{DOP}=\frac{\sqrt{Q^{2}+U^{2}+V^{2}}}{I},
$$

degree of linear polarization

$$
\mathrm{DOLP}=\frac{\sqrt{Q^{2}+U^{2}}}{I},
$$

and degree of circular polarization

$$
\mathrm{DOCP}=\frac{V}{I}
$$

Note that the degree of polarization of light should not exceed unity. This therefore imposes the following restriction on the Stokes parameters

$$
I \geq \sqrt{Q^{2}+U^{2}+V^{2}},
$$

where the equality and the inequality signs correspond to completely and partially polarized states, respectively. 
While the Stokes vectors represent the polarization state of light, a $4 \times 4$ matrix M, known as the Mueller matrix (after its inventor Hans Mueller in the 1940s) describes the transfer function of any medium in its interaction with polarized light: ${ }^{6,18-21}$

$$
\begin{aligned}
& \mathbf{S}_{\mathrm{o}}=\mathbf{M ~ \mathbf { S } _ { \mathrm { i } }} \\
& {\left[\begin{array}{c}
I_{o} \\
Q_{o} \\
U_{o} \\
V_{o}
\end{array}\right]=\left[\begin{array}{llll}
m_{11} & m_{12} & m_{13} & m_{14} \\
m_{21} & m_{22} & m_{23} & m_{24} \\
m_{31} & m_{32} & m_{33} & m_{34} \\
m_{41} & m_{42} & m_{43} & m_{44}
\end{array}\right]\left[\begin{array}{c}
I_{i} \\
Q_{i} \\
U_{i} \\
V_{i}
\end{array}\right]} \\
& =\left[\begin{array}{c}
m_{11} I_{i}+m_{12} Q_{i}+m_{13} U_{i}+m_{14} V_{i} \\
m_{21} I_{i}+m_{22} Q_{i}+m_{23} U_{i}+m_{24} V_{i} \\
m_{31} I_{i}+m_{32} Q_{i}+m_{33} U_{i}+m_{34} V_{i} \\
m_{41} I_{i}+m_{42} Q_{i}+m_{43} U_{i}+m_{44} V_{i}
\end{array}\right],
\end{aligned}
$$

with $\mathbf{S}_{\mathrm{i}}$ and $\mathbf{S}_{\mathbf{o}}$ being the Stokes vectors of the input and output light, respectively. The $4 \times 4$ real Mueller matrix M possesses at most 16 independent parameters (or 15 if the absolute intensity is excluded), including depolarization information. All the medium polarization properties are encoded in the various elements of the Mueller matrix, which can thus be thought of as the complete "optical polarization fingerprint" of a sample.

The fundamental requirement that real Mueller matrices must meet is that they map physical incident Stokes vectors into physically resultant Stokes vectors [satisfying Eq. (5)]. ${ }^{20,21}$ The conditions for physical realizability of $\mathbf{M}$, their associated interpretations, the relationships between the two formalisms (Jones and the Stokes-Mueller) have been discussed in the literature. ${ }^{20-22}$

Although both the Jones and Stokes-Mueller approaches rely on linear algebra and matrix formalisms, they are different in many aspects. Specifically, the Stokes-Mueller formalism has certain advantages. First of all, it can encompass any polarization state of light, whether it is natural, totally, or partially polarized (can thus deal with both polarizing and depolarizing optical systems). Secondly, the Stokes vectors and Mueller matrices can be measured with relative ease using intensity-measuring conventional (square-law detector) instruments, including most polarimeters, radiometers, and spectrometers. Since biological tissue is a turbid medium where significant depolarization is encountered due to strong multiple scattering effects, the Stokes-Mueller formalism has been used in most tissue polarimetry applications. In contrast, the use of the Jones formalism has been limited as a complementary theoretical approach to the Mueller matrix calculus, or to studies in clear media, specular reflections, and thin films where polarization loss is not an issue. In this paper, we review the use of the Stokes-Mueller approach for noninvasive assessment of biological tissues, discuss inverse analysis methods for extraction/quantification of the intrinsic tissue polarimetry characteristics, and provide selected illustrative application examples of tissue polarimetry. In the following, we define the ba- sic medium polarization properties through the Mueller matrix formalism.

\subsection{Basic Medium Polarimetry Characteristics}

\subsubsection{Depolarization}

If an incident state is $100 \%$ polarized and the exiting state has a degree of polarization less than unity, then the system is said to possess depolarization property. Depolarization is usually encountered due to multiple scattering of photons (although randomly oriented uniaxial birefringent domains can also depolarize light); incoherent addition of amplitudes and phases of the scattered field results in scrambling of the output polarization state. The general form of a pure depolarization Mueller matrix is $^{20,23}$

$$
\mathbf{M}_{\Delta}=\left(\begin{array}{llll}
1 & 0 & 0 & 0 \\
0 & a & 0 & 0 \\
0 & 0 & b & 0 \\
0 & 0 & 0 & c
\end{array}\right),|a|,|b|,|c| \leq 1 .
$$

Here $1-|a|$ and $1-|b|$ are depolarization factors for linear polarization (horizontal/vertical and $+45 \mathrm{deg} /-45 \mathrm{deg}$ linear polarizations, respectively) and $1-|c|$ is the depolarization factor for circular polarization. The net depolarization factor is usually defined as

$$
\Delta=1-\frac{|a|+|b|+|c|}{3}=1-\frac{\left|\operatorname{tr}\left(M_{\Delta}\right)-1\right|}{3}, 0 \leq \Delta \leq 1 \text {. }
$$

Note that this definition of depolarization factor of the medium is different from the conventional Stokes parameter-based definition of degree of polarization of the light [Eqs. (2)-(4)]. The latter represents the degree of polarization resulting from all depolarization effects encountered by the beam, and also depends on the incident Stokes vector. In contrast, the depolarization factors of Eq. (9) represent the pure depolarizing transfer function of the medium. Overall, the two can be roughly related via $\mathrm{DOP}_{\text {light }} \sim 1-\Delta_{\text {medium }}$ (and similarly for linear and circular states), but this is strictly an equality only in special cases. In general though, a medium with a high value of $\Delta$ is significantly depolarizing, and the degree of polarization of the light after interacting with it will be quite low.

\subsubsection{Retardance}

The next two polarization effects, retardance and diattenuation, arise from differences in refractive indices for different polarization states, and are often described in terms of ordinary and extraordinary indices and axes. Differences in the real parts of the refractive index lead to linear (circular) birefringence, whereas differences in the imaginary parts of the refractive index cause linear (circular) dichroism (which manifests itself as diattenuation, described below). Specifically, retardance is the phase shift between two orthogonal polarizations of the light. Linear retardance $\delta$ (birefringence) arises due to a difference in phase between orthogonal linear polarization states (between vertical and horizontal, or between $45 \mathrm{deg}$ and $-45 \mathrm{deg}$ ). Circular retardance $\psi$ (optical rotation) arises due to difference in phase between right circularly polarized (RCP) and left circularly polarized (LCP) states. The general form of a Mueller matrix of 
a pure linear retarder with retardance $\delta$ and fast axis oriented at an angle $\theta$ with respect to the horizontal is ${ }^{20,23}$

$$
\left(\begin{array}{cccc}
1 & 0 & 0 & 0 \\
0 & \cos ^{2} 2 \theta+\sin ^{2} 2 \theta \cos \delta & \sin 2 \theta \cos 2 \theta(1-\cos \delta) & -\sin 2 \theta \sin \delta \\
0 & \sin 2 \theta \cos 2 \theta(1-\cos \delta) & \sin ^{2} 2 \theta+\cos ^{2} 2 \theta \cos \delta & \cos 2 \theta \sin \delta \\
0 & \sin 2 \theta \sin \delta & -\cos 2 \theta \sin \delta & \cos \delta
\end{array}\right)
$$

Similarly, the Mueller matrix for a circular retarder with optical rotation value $\psi$ is ${ }^{20,23}$

$$
\left(\begin{array}{cccc}
1 & 0 & 0 & 0 \\
0 & \cos 2 \psi & -\sin 2 \psi & 0 \\
0 & \sin 2 \psi & \cos 2 \psi & 0 \\
0 & 0 & 0 & 1
\end{array}\right)
$$

\subsubsection{Diattenuation}

Diattenuation $(d)$ of an optical element corresponds to differential attenuation of orthogonal polarizations for both linear and circular polarization states. Accordingly, linear diattenuation is defined as differential attenuation of two orthogonal linear polarization states and circular diattenuation is defined as differential attenuation of RCP and LCP. Mathematically, the Mueller matrix for an ideal diattenuator can be defined using the parameters, $q$ and $r$, intensity transmittance (or reflectance) for the two incident orthogonal polarization states (either linear or circular), and the orientation angle of the principal axis $(\theta)$. Using this convention, the general Mueller matrix for a linear diattenuator is defined as $^{20,23}$

$$
\left(\begin{array}{cccc}
q+r & (q-r) \cos 2 \theta & (q-r) \sin 2 \theta & 0 \\
(q-r) \cos 2 \theta & (q+r) \cos ^{2} 2 \theta+2 \sqrt{(q r)} \sin ^{2} 2 \theta & (q+r-2 \sqrt{(q r)}) \sin 2 \theta \cos 2 \theta & 0 \\
(q-r) \sin 2 \theta & (q+r-2 \sqrt{(q r)}) \sin 2 \theta \cos 2 \theta & (q+r) \cos ^{2} 2 \theta+2 \sqrt{(q r)} \sin ^{2} 2 \theta & 0 \\
0 & 0 & 0 & 2 \sqrt{(q r)}
\end{array}\right) .
$$

Similarly for circular diattenuation, the general form of the Mueller matrix is ${ }^{20,23}$

$$
\left(\begin{array}{cccc}
q+r & 0 & 0 & q-r \\
0 & 2 \sqrt{(q r)} & 0 & 0 \\
0 & 0 & 2 \sqrt{(q r)} & 0 \\
q-r & 0 & 0 & q+r
\end{array}\right)
$$

The simplest form of a diattenuator is the ideal polarizer that transforms incident unpolarized light to completely polarized light ( $d=1$ for ideal polarizer), although often with a significant reduction in the overall intensity $I$. Note that diattenuation is analogous to dichroism, which is defined as the differential absorption of two orthogonal linear polarization states (linear dichroism) or RCP - LCP (circular dichroism), but is more general in a sense that it is defined in terms of differential attenuation (either by absorption or scattering).

\section{Experimental Tissue Polarimetry Systems}

As outlined in Sec. 1, biomedical polarimetry research has two major directions, tissue imaging and tissue characterization.
First, polarization can be used as an effective tool to discriminate against multiply scattered light and thus can facilitate higher resolution imaging of tissue and its underlying structure. ${ }^{17}$ Moreover, the intrinsic polarimetry characteristics themselves contain a wealth of morphological, biochemical, and functional information that can be exploited for noninvasive and quantitative tissue diagnosis. ${ }^{15,16,24}$ For either of these applications, accurate measurement of the polarization retaining signal is extremely important. In this regard, many of the traditional polarimetry systems are not suitable for biological tissue examination (e.g., crossed linear polarizers used in microscopy for examining thin fixed ex vivo tissue slices). This follows because multiple scattering in thick tissues leads to depolarization of light, creating a large depolarized source of noise that hinders the detection of the small remaining information-carrying polarization signal. A variety of experimental tools have therefore been developed to maximize measurement sensitivity, so that reliable measurements and analyses of the tissue polarimetry data can be performed. These methods can be employed to perform measurement of both Stokes vector of the light upon interacting with the sample, and/or of the Mueller matrix of the sample itself, as described below. 


\subsection{Stokes Vector Polarimeters}

As mentioned in Sec. 2.2, the four Stokes parameters of light can be determined by performing six intensity measurements involving linear and circular polarization states $\left(I_{H}, I_{V}, I_{P}, I_{M}, I_{R}\right.$, and $\left.I_{L}\right) .{ }^{21}$ Alternatively, this can be achieved by just four measurements, exploiting the property $\left(I_{H}+I_{V}=I_{P}+I_{M}=I_{L}\right.$ $\left.+I_{R}\right) .^{25}$ In this approach, a circular polarizer is designed consisting of a linear polarizer whose transmission axis is set at $+45 \mathrm{deg}$ with respect to the horizontal direction, followed by a quarter wave-plate with its fast axis parallel to the horizontal direction. Three sets of intensity measurements $\left[I_{\mathrm{Cir}}(\alpha)\right]$ are performed by changing the angle $(\alpha)$ of this circular polarizer to 0,45 , and $90 \mathrm{deg}$ with respect to the horizontal axis. The combined polarizer is then flipped to the other side and the final intensity measurement $\left[I_{\operatorname{Lin}}(\alpha)\right]$ is made by setting $\alpha$ at $0 \mathrm{deg}$. The four Stokes parameters can be determined from the measured intensities as

$$
\left[\begin{array}{c}
I \\
Q \\
U \\
V
\end{array}\right]=\left[\begin{array}{c}
I_{c i r}(0 \mathrm{deg})+I_{c i r}(90 \mathrm{deg}) \\
I-2 I_{c i r}(45 \mathrm{deg}) \\
I_{c i r}(0 \mathrm{deg})-I_{c i r}(90 \mathrm{deg}) \\
-I+2 I_{L i n}(0 \mathrm{deg})
\end{array}\right] .
$$

This particular scheme of measurement was originally proposed by Collett. ${ }^{25}$ Although this method has been employed in some experimental depolarization studies to measure Stokes parameters of light transmitted (or backscattered) from tissue and tissue-like turbid media, ${ }^{26-30}$ a more sensitive detection scheme is desirable, specifically in applications involving accurate quantification of the intrinsic tissue polarimetry characteristics. ${ }^{15,16}$ One possible method for improving the sensitivity of the measurement procedure is the use of polarization modulation with synchronous detection. Many sensitive detection schemes are possible with this approach. ${ }^{31-36}$ Some of these perform polarization modulation on the light that is incident on the sample; others modulate the sample-emerging light, by placing the polarization modulator between the sample and the detector. The resultant signal can be analyzed to yield sample-specific polarization properties that can then be linked to the quantities of interest.

By way of illustration, a schematic of the experimental polarimetry system employing polarization modulation and synchronous lock-in-amplifier detection is shown in Fig. 1(a). ${ }^{31}$ Unpolarized light from a laser is used to seed the system. The light first passes through a mechanical chopper operating at a frequency $f_{\mathrm{c}} \sim 500 \mathrm{~Hz}$; this is used in conjunction with lock-in amplifier detection to accurately establish the overall signal intensity levels. The input optics [a linear polarizer $P_{1}$ with/without the quarter wave-plate $\left.\left(\mathrm{QWP}_{1}\right)\right]$ enables generation of any of the four input polarization states, $0 \mathrm{deg}$ (Stokes vector $\left.\left[\begin{array}{llll}1 & 1 & 0 & 0\end{array}\right]^{\mathrm{T}}\right), 45 \mathrm{deg}$ (Stokes vector $\left[\begin{array}{llll}1 & 0 & 1 & 0\end{array}\right]^{\mathrm{T}}$ ), and $90 \mathrm{deg}$ (Stokes vector $\left[\begin{array}{llll}1 & -1 & 0 & 0\end{array}\right]^{\mathrm{T}}$ ) linear polarizations, as well as circular polarization (Stokes vector $\left[\begin{array}{llll}1 & 0 & 0 & 1\end{array}\right]^{\mathrm{T}}$ ) incident on the sample. Following light-tissue interactions, the detection optics begin with a removable quarter wave-plate $\left(\mathrm{QWP}_{2}\right)$ with its fast axis oriented at $-45 \mathrm{deg}$, when in place allowing for the measurement of Stokes parameters $Q$ and $U$ (a)

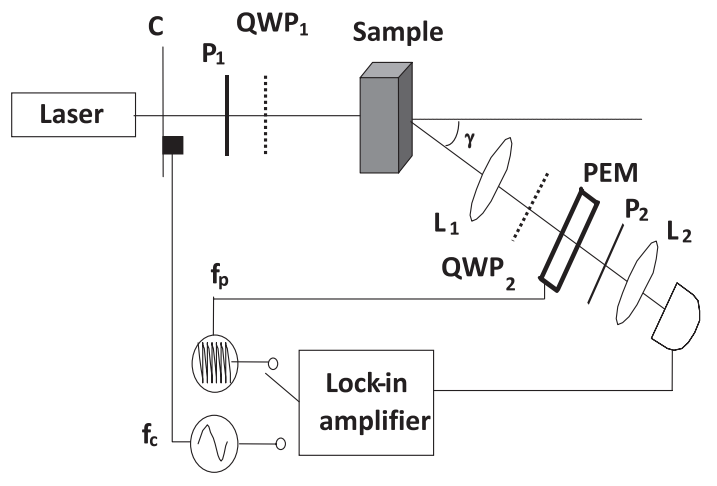

(b)

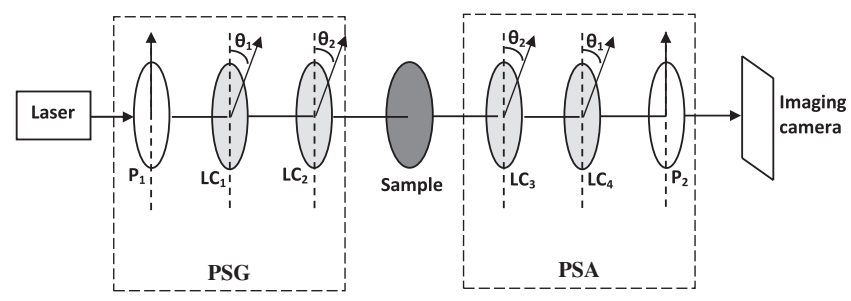

Fig. 1 (a) A schematic of the experimental polarimetry system employing polarization modulation and synchronous lock-in-amplifier detection. $C$, mechanical chopper; $\mathrm{P}_{1}, \mathrm{P}_{2}$, polarizers; $\mathrm{QWP} \mathrm{P}_{1}, \mathrm{QWP}_{2}$, removable quarter wave-plates; $\mathrm{L}_{1}, \mathrm{~L}_{2}$ lenses; PEM, photoelastic modulator; APD, photodetector; $f_{C}, f_{p}$ modulation frequencies of mechanical chopper and PEM, respectively. The detection optics can be rotated by an angle $\gamma$ around the sample. (Adopted from Ref. 16,) (b) A schematic of the liquid crystal variable retarder polarimeter. $\mathrm{P}_{1}, \mathrm{P}_{2}$, linear polarizers; $\mathrm{LC}_{1}, \mathrm{LC}_{2}, \mathrm{LC}_{3}, \mathrm{LC}_{4}$, liquid crystal variable retarders. $\mathrm{P}_{1}, \mathrm{LC}_{1}$ (with retardance of $\delta_{1}$ having orientation angle $\theta_{1}$ ), LC 2 (with retardance of $\delta_{2}$ having orientation angle $\theta_{2}$ ) form the (PSG) unit. LC $_{3}$ (with retardance of $\delta_{2}$ having orientation angle $\theta_{2}$ ) LC 4 (with retardance of $\delta_{1}$ having orientation angle $\theta_{1}$ ), and $P_{2}$ form the (PSA) unit. The schematic is shown for transmission measurements; other detection geometries are also possible using this scheme.

(linear polarization descriptors), and when removed allowing for the measurement of Stokes parameter $V$ (circular polarization descriptor). The tissue-scattered light then passes through a photoelastic modulator (PEM), which is a linearly birefringent resonant device operating in the kilohertz range (e.g., $f_{\mathrm{p}}$ $=50 \mathrm{kHz}$ ). The fast axis of the PEM is at 0 deg and its retardation is modulated according to the sinusoidal function $\delta_{\text {PEM }}(t)$ $=\delta_{\mathrm{o}} \sin \omega t$, where $\omega_{\mathrm{p}}=2 \pi f_{\mathrm{p}}$ and $\delta_{\mathrm{o}}$ is the user-specified amplitude of maximum retardation of PEM. The light finally passes through a linear analyzer orientated at $45 \mathrm{deg}$, converting the PEM-imparted polarization modulation to an intensity modulation suitable for photodetection. The resulting modulated intensity is collected using a pair of lenses and is relayed to an avalanche photodiode detector. The detected signal is sent to a lock-in amplifier with its reference input toggling between the frequencies of the chopper $(500 \mathrm{~Hz})$ and the PEM controller $(50 \mathrm{kHz}$ and harmonics) for synchronous detection of their respective signals. 
For this particular experimental arrangement, the Stokes vector of light after the analyzing block $\left[I_{f} Q_{f} U_{f} V_{f}\right]^{\mathrm{T}}$ can be related to that of the sample-emerging beam $[I Q U V]^{\mathrm{T}}$ (with detection quarter wave-plate in place) using Mueller matrix algebra ${ }^{31}$

$$
\left(\begin{array}{c}
I_{f} \\
Q_{f} \\
U_{f} \\
V_{f}
\end{array}\right)=\frac{1}{2}\left(\begin{array}{cccc}
1 & 0 & 1 & 0 \\
0 & 0 & 0 & 0 \\
1 & 0 & 1 & 0 \\
0 & 0 & 0 & 0
\end{array}\right) \quad\left(\begin{array}{cccc}
1 & 0 & 0 & 0 \\
0 & 1 & 0 & 0 \\
0 & 0 & \cos \delta & \sin \delta \\
0 & 0 & -\sin \delta & \cos \delta
\end{array}\right) \quad\left(\begin{array}{cccc}
1 & 0 & 0 & 0 \\
0 & 0 & 0 & 1 \\
0 & 0 & 1 & 0 \\
0 & -1 & 0 & 0
\end{array}\right)\left(\begin{array}{l}
I \\
Q \\
U \\
V
\end{array}\right),
$$

and when the detection quarter wave-plate is removed as,

$$
\left(\begin{array}{c}
I_{f r} \\
Q_{f r} \\
U_{f r} \\
V_{f r}
\end{array}\right)=\frac{1}{2}\left(\begin{array}{cccc}
1 & 0 & 1 & 0 \\
0 & 0 & 0 & 0 \\
1 & 0 & 1 & 0 \\
0 & 0 & 0 & 0
\end{array}\right)\left(\begin{array}{cccc}
1 & 0 & 0 & 0 \\
0 & 1 & 0 & 0 \\
0 & 0 & \cos \delta & \sin \delta \\
0 & 0 & -\sin \delta & \cos \delta
\end{array}\right)\left(\begin{array}{c}
I \\
Q \\
U \\
V
\end{array}\right) .
$$

The detected time dependent intensities are

$$
I_{f}(t)=\frac{I}{2}[1-q \sin \delta+u \cos \delta],
$$

and

$$
I_{f r}(t)=\frac{I}{2}[1-v \sin \delta+u \cos \delta] .
$$

Here $q=Q / I, u=U / I$, and $v=V / I$, and $\delta$ is the time-dependent PEM retardation, $\delta=\delta_{0} \sin \omega t$. By expanding the time varying parts of Eqs. (17) and (18) in Fourier series of Bessel functions, and by setting the peak retardance of the PEM $\delta_{0}=2.405$ radians (the retardance value at which the zeroth-order Bessel function exhibits its first null), one can relate the normalized Stokes parameters to the synchronously-detected signals at the chopper frequency $V_{1 f \mathrm{c}}$ (the dc signal level), and at the first and second harmonics of the PEM frequency $V_{1 f \mathrm{p}}$ and $V_{2 f \mathrm{p}}$. When the detection quarter wave-plate in place, this yields ${ }^{31}$

$$
\begin{aligned}
& q=\frac{V_{1 f p}}{\sqrt{2} J_{1}\left(\delta_{o}\right) V_{1 f c}}, \\
& u=\frac{V_{2 f p}}{\sqrt{2} J_{2}\left(\delta_{o}\right) V_{1 f c}},
\end{aligned}
$$

and when the detection quarter wave-plate is removed

$$
v=\frac{V_{1 f p}}{\sqrt{2} J_{1}\left(\delta_{o}\right) V_{1 f c}} .
$$

The synchronous lock-in detected signals at the first and second harmonics of PEM frequency, and that detected at the chopper frequency, can thus be used to determine the Stokes parameters with increased signal-to-noise (SNR) for any given incident state of polarization [P1 and QWP1 combinations in Fig. 1(a)]. ${ }^{31}$

\subsection{Mueller Matrix Polarimeters}

With some additional measurements and analysis, one can go beyond polarimetric light description (Stokes vectors) and determine the polarization transfer function of the sample itself (Mueller matrix). For $4 \times 4$ Mueller matrix determination, both dc (involving sequential static measurements) and ac modulation-based measurement procedures have been employed. In fact, the polarization modulation approach described in Sec. 3.1 can also perform sensitive Mueller polarimetry. This can be achieved by sequentially cycling the input polarization between four states (linear polarization at $0 \mathrm{deg}, 45 \mathrm{deg}, 90 \mathrm{deg}$, and right circular polarization) and by measuring the output Stokes vector for each respective input states. The elements of the resulting four measured Stokes vectors (16 values) can be algebraically manipulated to solve for the sample Mueller matrix: $:^{37}$

$$
\mathbf{M}(i, j)=\left(\begin{array}{cccc}
\frac{1}{2}\left(I_{H}+I_{V}\right) & \frac{1}{2}\left(I_{H}-I_{V}\right) & I_{p}-\mathbf{M}(1,1) & I_{R}-\mathbf{M}(1,1) \\
\frac{1}{2}\left(Q_{H}+Q_{V}\right) & \frac{1}{2}\left(Q_{H}-Q_{V}\right) & Q_{p}-\mathbf{M}(2,1) & Q_{R}-\mathbf{M}(2,1) \\
\frac{1}{2}\left(U_{H}+U_{V}\right) & \frac{1}{2}\left(U_{H}-U_{V}\right) & U_{p}-\mathbf{M}(3,1) & U_{R}-\mathbf{M}(3,1) \\
\frac{1}{2}\left(V_{H}+V_{V}\right) & \frac{1}{2}\left(V_{H}-V_{V}\right) & V_{p}-\mathbf{M}(4,1) & V_{R}-\mathbf{M}(4,1)
\end{array}\right) .
$$


Here, the four input states are denoted with the subscripts $H$ (0 deg), $P$ (45 deg), $V$ (90 deg), and $R$ (right circularly polarized; left circular incidence can be used as well, resulting only in a sign change). The indices $i, j=1,2,3,4$ denote rows and columns, respectively.

The described experimental embodiment of the polarization modulation/synchronous detection approach has been used by us for both Stokes vector and Mueller matrix measurements in complex tissue-like turbid media and in actual tissues, ${ }^{37,38}$ some of the results are presented subsequently in this paper.

Among the various other modulation-based Mueller matrix polarimeters, the dual rotating retarder approach has been widely used in tissue polarimetry investigations. ${ }^{39-41}$ In this scheme, polarization modulation of the incident state is generated by passing light first through a fixed linear polarizer and then through a rotating linear retarder (retardation $\delta_{1}$ ) with angular speed $\omega_{1}$. The analyzing optics contains another rotating retarder (retardation $\delta_{2}$, synchronously rotating at angular speed $\omega_{2}$ ) and a fixed linear polarizer. In the usual configuration, the retardation values of the two retarders are chosen to be the same $\delta_{1}=\delta_{2}$ $=\pi / 2$, the axis of the polarizer and the analyzer are kept parallel, and the angular rotation speeds of the retarders are kept as $\omega_{1}=\omega$ and $\omega_{2}=5 \omega$, respectively. ${ }^{39,41}$ The rotation of the retarders at these different rates results in a modulation of the detected intensity signal, as can be understood by sequentially writing the Mueller matrices corresponding to each optical element (polarizers, retarders, and the sample). Note that for this specific scheme, the modulation in the detected intensity arises due to harmonic variation of the orientation angle of the two retarders kept at the polarizing and analyzing end of the polarimeter ( $\theta$ and $5 \theta$, respectively). It has been shown that the five to one ratio of angular rotation speeds of the two retarders encodes all 16 Mueller matrix elements onto the amplitude and phases of 12 frequencies in the detected intensity signal. The detected signal is Fourier analyzed and the Mueller matrix elements are constructed from the Fourier coefficients. A more general approach based on this scheme may employ arbitrary values for linear retardations $\left(\delta_{1}\right.$ and $\left.\delta_{2}\right)$, rotation speed ratios $\left(\omega_{1} / \omega_{2}\right)$, and axis of the linear polarizers, depending upon which elements (rows/columns) of Mueller matrix are given a priority in terms of higher determination precision and/or SNR. ${ }^{39,41}$
Snapshot Mueller matrix polarimeter is another important development in modulation-based Mueller matrix polarimetry. ${ }^{42}$ The approach exploits wavelength polarization coding and decoding for high sensitivity, instantaneous measurement of all 16 Mueller matrix elements simultaneously. Briefly, several wavelength-encoded polarization states are generated with a broadband spectrum source, two birefringent retarders, and a linear polarizer. The wavelength decoding is performed using a similar combination of birefringent retarders and a linear polarizer. The thickness of the retarders in the encoding (polarization state generator) and decoding (polarization state analyzer) systems are optimized to generate and analyze sufficient number of polarization states; the resulting spectral signals (over a narrow spectral range $\Delta \lambda \sim 10 \mathrm{~nm}$ ) recorded using a spectrometer are Fourier analyzed to yield all 16 Mueller matrix elements.

Note that the polarization modulation-based polarimeters described above are convenient for point polarimetry measurements; large area imaging is generally not feasible using these approaches (since these typically employ a synchronous detection scheme). Such imaging polarimetry can be accomplished by dc measurements involving sequential measurements with different combinations of source polarizers and detection analyzers, albeit with lower SNR than the synchronous ac detection schemes described above. Because a general $4 \times 4$ Mueller matrix has 16 independent elements, at least 16 independent measurements are required; ${ }^{43}$ due to the low sensitivity of the dc approach, alternatives have been investigated. For example, polarimetric imaging systems based on liquid crystal variable retarders enable measurement of the Mueller matrix elements with higher sensitivity and precision. ${ }^{44-46}$ A schematic of such a measurement strategy is shown in Fig. 1(b). The polarimetry system is comprised of a polarization state generator (PSG) and a polarization state analyzer (PSA) unit coupled to an imaging camera for spatially resolved signal detection. The PSG consists of a linear polarizer $\left(P_{1}\right)$ and two liquid crystal variable retarders $\left(\mathrm{LC}_{1}\right.$ and $\mathrm{LC}_{2}$ having variable retardance of $\delta_{1}$ and $\delta_{2}$, respectively). Generally, the birefringence axes of $\mathrm{LC}_{1}$ and $\mathrm{LC}_{2}$ are kept at angles $\theta_{1}$ and $\theta_{2}$, respectively with respect to the axis of the polarizer $P_{1}{ }^{45}$ In a more specific arrangement (which has been widely used), the angles $\theta_{1}$ and $\theta_{2}$ are chosen to be 45 and $0 \mathrm{deg}$, respectively. The Stokes vector generated from this arrangement and incident on the sample is ${ }^{46}$

$$
\left(\begin{array}{l}
I_{f} \\
Q_{f} \\
U_{f} \\
V_{f}
\end{array}\right)=\left(\begin{array}{cccc}
1 & 0 & 0 & 0 \\
0 & 1 & 0 & 0 \\
0 & 0 & \cos \delta 2 & \sin \delta 2 \\
0 & 0 & -\sin \delta 2 & \cos \delta 2
\end{array}\right)\left(\begin{array}{cccc}
1 & 0 & 0 & 0 \\
0 & \cos \delta 1 & 0 & -\sin \delta 1 \\
0 & 0 & 1 & 0 \\
0 & \sin \delta 1 & 0 & \cos \delta 1
\end{array}\right)\left(\begin{array}{l}
1 \\
1 \\
0 \\
0
\end{array}\right)=\left(\begin{array}{c}
1 \\
\cos \delta 1 \\
\sin \delta 2 \sin \delta 1 \\
\cos \delta 2 \sin \delta 1
\end{array}\right)
$$

As is obvious from Eq. (23), any possible state of polarization can be generated by selectively varying $\delta_{1}$ and $\delta_{2}$. The PSA unit also consists of similar arrangements of liquid crystal variable retarders $\left(\mathrm{LC}_{3}\right.$ and $\left.\mathrm{LC}_{4}\right)$ and linear polarizer, but positioned in reverse order, and followed by a detector (for imaging applica- tions, this is a CCD camera). The polarimetry signal analysis proceeds as follows.

The PSG output can be represented by $\mathbf{W}$, a $4 \times 4$ matrix whose column vectors are the four generated Stokes vectors $\mathbf{S}_{\mathbf{i}}$ incident on the sample. Similarly, after sample interactions, 
the PSA results can also be described by a $4 \times 4$ matrix A. The Stokes vectors of light to be analyzed are projected on four basis states that are the row vectors of the $4 \times 4$ analysis matrix A. For the construction of the Mueller matrix, a sequence of 16 measurements are performed. This $4 \times 4$ intensity measurement matrix $M_{i}$ can be written as ${ }^{20,47}$

$$
\mathbf{M}_{\mathbf{i}}=\mathbf{A} \cdot \mathbf{M} . \mathbf{W}
$$

where $\mathbf{M}$ is the sample Mueller matrix, which when presented as $1 \times 16$ vector $\left(\mathbf{M}_{\mathbf{v e c}}\right)$, can be related to the intensity measurement $(1 \times 16)$ vector $\mathbf{M}_{\mathbf{i v e c}}$ as

$$
\mathbf{M}_{\text {ivec }}=\mathbf{Q} \cdot \mathbf{M}_{\mathrm{vec}}
$$

where $\mathbf{Q}$ is a $16 \times 16$ matrix given as Kronecker product of $\mathbf{A}$ with transpose of $\mathbf{W}$

$$
\mathbf{Q}=\mathbf{A} \otimes \mathbf{W}^{\mathrm{T}}
$$

Note that this type of construction of the Mueller matrix allows for calibration of the actual values of the PSG and PSA matrices ( $\mathbf{A}$ and $\mathbf{W}) .{ }^{47}$ Based on this approach, several measurement schemes are possible. In fact, the choice of the values for retardance $\delta_{1}$ and $\delta_{2}$, the orientation angles of the retarders with respect to the polarizers (analyzers) can be optimized to minimize the noise in the resulting Mueller matrix M. Such optimized measurement strategies have also been explored for performing Mueller matrix measurements in tissues. ${ }^{45}$

Recently, a novel stroboscopic illumination technique has been explored to facilitate large area imaging using a polarization modulation scheme. ${ }^{48}$ This approach utilizes a pulsed laser diode to illuminate the object. The short current pulses of this laser diode are precisely controlled by a programmable pulse generator. The temporal reference is triggered by the controller of a PEM operating at $50 \mathrm{kHz}$. This synchronization procedure facilitates freezing of the intensity variation of the PEM modulated signal at desirable temporal phases. Measurement of the intensity (using a CCD camera) at four specific temporal phases (frozen via stroboscopic illumination) are used to deduce the two-dimensional images of the two well-known ellipsometric parameters. ${ }^{48,49}$ Note, however, this approach has not yet been explored for complete (16 element) Mueller matrix imaging.

Having described the various experimental strategies for sensitive measurement of Stokes vector and Mueller matrix in turbid medium-like tissue, we now turn to another challenging problem of accurately modeling the polarization signals in turbid media, in the forward sense.

\section{Modeling Polarized Light Transport in Complex Turbid Media}

To aid tissue polarimetry in successful implementation of polarization gated optical imaging and for quantitative determination of intrinsic tissue polarimetry characteristics, accurate forward modeling is enormously useful. This helps in gaining physical insight, designing, and optimizing experiments, and analyzing/interpreting the measured data. The use of electromagnetic theory with Maxwell's equations is the most rigorous and bestsuited method for polarimetry analysis, at least in clear media with well-defined optical interfaces. However, unlike optically clear media, tissue is a turbid medium possessing microscopically inhomogeneous complex dielectric structures (macro- molecules, cell organelles, organized cell structures, blood and lymphatic networks, extra-cellular matrix, interstitial layers, etc.). Due to the ensuing complexity, the Maxwell's equations approach for polarized light propagation in such a complex turbid medium is impractical and is not presently feasible. ${ }^{11,50}$ Instead, light propagation through such media is often modeled using the radiative transport theory. ${ }^{11,50,51}$ Although the scalar radiative transport theory and its simplified approximation, the diffusion equation, has been successfully used to model light transport in tissue (specifically light intensity distribution in tissue volume, diffuse reflectance, etc.), both are intensitybased techniques, and hence typically neglect polarization. ${ }^{51}$ Alternatively, the vector radiative transfer equation (VRTE), which includes polarization information by describing transport of the Stokes vectors of light (photon packet) through a random medium, ${ }^{11,51}$ has been explored for tissue polarimetry modeling. However, solving the VRTE in real systems is rather complex. A wide range of analytical and numerical techniques have been developed to solve VRTE, namely, the small angle approximation, the transfer matrix, the singular eigenfunction, the adding-doubling, the discrete ordinates, the successive orders, and the invariant embedding methods. ${ }^{11,51}$ Unfortunately, these are often too slow and insufficiently flexible to incorporate the necessary boundary conditions for arbitrary geometries and arbitrary optical properties as desirable in case of tissue. A more general and robust approach is the Monte Carlo (MC) technique, as described in greater detail below. First, we present a brief overview of some of the simpler analytical approximations developed to deal with depolarization of multiply scattered waves in turbid medium. These are useful for understanding the mechanisms of depolarization of light in turbid media, for performing rough estimates and order-of-magnitude calculations, and for optimizing the polarization gating schemes for tissue imaging.

\subsection{Modeling Depolarization of Multiply Scattered Light in Turbid Medium: Approximate Analytical/Heuristic Approaches}

Various methods using photon diffusion formalisms, random walk models, maximum entropy principles, etc., have been explored for modeling of depolarization of multiply scattered waves in random medium. ${ }^{52-59}$ The aim of these models have been to derive analytical relationships between various quantities of practical interest such as the degree of polarization (either linear or circular) of forward- or backscattered light from a turbid medium, average pathlengths, the optical transport parameters of the medium, and so forth. As in the case for radiative transport theory, in these models also, the turbid medium is considered to have bulk-average scattering and absorption properties, representative of isotropic tissue volumes. For this description, the turbid medium is usually modeled through the optical transport parameters, namely, the absorption coefficient $\left(\mu_{a}\right)$, single scattering coefficient $\left(\mu_{\mathrm{s}}\right)$, and single scattering anisotropy $(g){ }^{50,51}$ As is known from the transport theory, the linear isotropic optical coefficients are defined so that $l_{a}=\mu_{a}^{-1}$ and $l_{s}=\mu_{s}^{-1}$ give the absorption and scattering mean free paths, respectively. The anisotropy parameter $g$ is defined as the average cosine of scattering angle. The value of $g$ ranges from -1 to +1 , where $g=-1$ corresponds to fully backward scattering, $g=0$ 
corresponds to forward-backward symmetric scattering, and $g$ $=+1$ corresponds to fully forward scattering. In general, the value of $g$ depends on the average size of the scatterers in the medium relative to the wavelength of the irradiation. For a medium comprised of scatterers whose size is much smaller than the wavelength (radius $a \ll \lambda$ ), anisotropy parameter $g$ is $\sim 0$, its value approaching unity $(g \sim 1)$ for medium comprised of larger sized scatterers $(a \geq \lambda)$. The latter regime applies to most biological tissues in the visible/near-infrared (NIR) spectral range. ${ }^{50}$ The other optical transport parameter frequently used in tissue optics is the reduced scattering coefficient $\mu_{s}^{\prime}=\mu_{s}(1-g) .^{50,51}$ This is relevant in the multiple scattering regime and its use assumes that the light intensity metrics (reflectance, transmittance, fluence) of a turbid volume with optical parameters $\mu_{a}, \mu_{s}$, and $g(\neq 0)$ are the same as those for an analogous volume with optical parameters $\mu_{a}, \mu_{s}^{\prime}$ and $g=0 .^{50}$ The corresponding mean free path, known as the transport mean free path is defined as $l^{*}$ $=\left(\mu_{s}^{\prime}\right)^{-1}$, and is referred to as the typical length scale over which the propagation direction of photons get randomized in a multiply scattering medium. Note that for forward-scattering media such as most biological tissues, $\mu_{s}^{\prime}<\mu_{s}$ by a factor of $(1-g)$, thus the transport mean free path $l^{*}$ is longer than the mean free path $l$ (by a factor of $1 /(1-g)$, for example $10 \times$ for $g=0.9)$.

Pioneering work in turbid polarimetry modeling was carried out by Bicout et al. ${ }^{53}$ They related the depolarization of light by multiple scattering to a process of entropy production. Based on the so-called maximum entropy principle, the single path (photon undergoing successive scattering events) degree of polarization decays exponentially with an increasing number of scattering events $(n) .{ }^{52,53}$ For a medium comprised of a collection of nonabsorbing, optically inactive, spatially uncorrelated spherical particles whose size is much smaller compared to wavelength (radius $a \ll \lambda, g \sim 0$, the so-called Rayleigh regime), expression for single path degree of linear and circular polarization (for incident linearly and circularly polarized light, respectively) can be expressed as ${ }^{53}$

$$
P_{L}(n)=\frac{3}{2} \exp [-n(l / \xi l)] \quad \text { and } \quad P_{C}(n)=\frac{3}{2} \exp [-n(l / \xi c)]
$$

Here, $l$ is the scattering mean free path (note that $g \sim 0$ here, there is no distinction between $l$ and $l^{*}$ ), and the parameters $\xi_{l}$ and $\xi_{c}$ are known as characteristic length scale of depolarization of incident linearly and circularly polarized light, respectively. For a medium comprised of ensemble of Rayleigh scatterers the values for $\xi_{l}$ and $\xi_{c}$ have the following approximate analytical form $^{53}$

$$
\xi_{l}=\frac{l}{\ln (10 / 7)} \quad \text { and } \quad \xi_{c}=\frac{l}{\ln (2)}, \quad \text { thus } \xi_{l} \cong 2 \xi_{c} .
$$

The results imply that circularly polarized light depolarizes $\sim 2 \times$ faster that linearly polarized light in a Rayleigh-scattering regime, at least for the commonly examined geometry of transmission through a tissue slab. The characteristic length scales of depolarization $\left(\xi_{l}\right.$ and $\xi_{c}$ ) are known to depend strongly on the size of the scatterers present in the medium (thus on the value of $g) .{ }^{53}$ In fact, empirical relationships between $\xi_{l}, \xi_{c}$, and $g$ has also been obtained based on random walk models, ${ }^{58}$ radiative transfer theory, ${ }^{55}$ and extended photon diffusion approximation combined with experimental measurements using diffusing wave spectroscopy (measurements of intensity fluctuations of light scattered from turbid media). ${ }^{57}$ This dependence can be summarized as follows:

$$
\frac{\xi_{l}}{l}=\frac{\frac{1}{\ln (10 / 7)}-2.5 g}{(1-g)} \quad \text { and } \quad \frac{\xi_{c}}{l}=\frac{\frac{1}{\ln 2}}{(1-g)} .
$$

The degree of residual polarization of multiply scattered light at a chosen detection point can be obtained by averaging (or weighing) the single path degree of polarization [Eq. (27)] over the pathlength distribution function $[\rho(n)]$ as

$$
P_{L, C}=\frac{\int_{0}^{\infty} P_{L, C}(n) \rho(n) d n}{\int_{0}^{\infty} \rho(n) d n} .
$$

This is conveniently done by using the approximate analytical expressions for $\rho(n)$ obtained from the solution of photon diffusion equation for a given detection geometry. ${ }^{50,51}$ The resulting degree of polarization of multiply scattered light transmitted or reflected from a slab of turbid medium can be expressed as follows.

Forward scattering geometry, transmission through a slab of thickness $L$ (Ref. 53)

$$
P_{L, C} \cong \frac{L}{l} \sinh \left[\frac{l}{\varsigma_{l, c}}\right] \exp \left[-\frac{L}{S_{l, c}}\right],
$$

where

$$
\varsigma_{l, c}=\left(\frac{3 \xi_{l, c}}{3}\right)^{1 / 2}
$$

Back-scattering geometry, reflection from a semi-infinite medium, $L \rightarrow \infty$ (Refs. 11 and 59)

$$
P_{L, C} \cong \frac{3}{2} \exp \left[-\gamma \sqrt{\frac{3 l^{*}}{\xi_{l, c}}}\right] .
$$

Here $l^{*}$ is the transport mean free path and $\gamma$ is the correlation decay parameter with its value ranging between from 1.5 to 3 . $^{11}$

The effect of medium absorption can also be modeled by modifying $\rho(n)$ to account for absorption-induced photon loss $\left\{\right.$ multiplying $p(n)$ by a factor exp $\left.\left[-\mu_{a} n\right]\right\}$. Accordingly, the expression for residual degree of polarization of light backscattered from an absorbing turbid medium takes the form ${ }^{11}$

$$
P_{L, C} \cong \frac{3}{2} \exp \left[-\gamma\left\{\sqrt{\frac{3 l^{*}\left(1+\mu_{a} \xi l\right)}{\xi_{l, c}}}\right\}-\sqrt{3 l^{*} \mu a}\right] .
$$

An important feature that can be noticed from Eqs. (28) and (29) [and thus in the resulting Eqs. (31) and (32)], is that for a medium comprised of Rayleigh scatterers $(a \ll \lambda, g \sim 0)$, depolarization of circularly polarized light is stronger than linearly polarized light $\left(\xi l>\xi_{c}\right)$. The reverse is the case $\left(\xi_{l}<\xi_{c}\right)$ for media comprised of larger scatterers ( $a \geq \lambda, g \geq 0.7$, the so-called Mie regime). This is illustrated in Fig. 2, where the computed variations of the length scales of depolarization $\left(\xi_{l}\right.$ and $\left.\xi_{c}\right)$ are shown as a function of size parameter of scatterer $(X)$ present in the medium. Here, $X=2 \pi a n_{m} / \lambda$ is the size parameter for scatterer of radius a (varying between 0.01 to $1.11 \mu \mathrm{m}$ ) having a refractive index $n_{s}$ (chosen to be 1.59 for the calculation) 


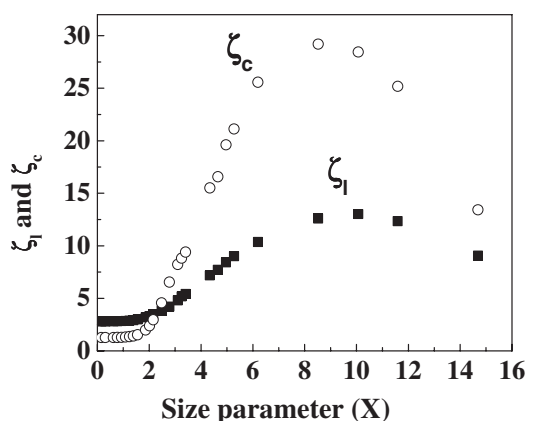

Fig. 2 The theoretically computed variation of length scales of depolarization for incident linearly $\left(\xi_{l}\right)$ and circularly $\left(\xi_{c}\right)$ polarized light as a function of size parameter of scatterer $(X)$. The values for $\xi_{l}$ and $\xi_{c}$ increase with increasing value of $X$, indicating weaker depolarization with increasing size of the scatterer. The general decreasing trend beyond a size parameter value of $X \geq 10$ has been attributed to Mie resonance effects (Ref. 53). See text for details.

embedded in a surrounding medium with refractive index $n_{m}$ $(=1.33) .{ }^{19}$ The wavelength of light was chosen to be symbol $\lambda$ $=0.6328 \mu \mathrm{m}$. The values for $\xi_{l}$ and $\xi_{c}$ are observed to increase with increasing value of $X$, indicating weaker depolarization with increasing size of the scatterer. Further, as can be seen, at lower value of size parameter $(X<2), \xi_{l}$ is larger than $\xi_{c}$ (depolarization of circular polarization is stronger as compared to depolarization of linear polarization) and reverse is the case for larger size parameter values $(X>2)$. These theoretical trends (of Fig. 2) have been confirmed by experimental depolarization studies conducted on turbid media comprised of spherical scatterers having varying sizes. ${ }^{26-30}$ The reason for the observed size parameter dependence of depolarization of light in turbid medium and the differences in relative rate of depolarization of linear and circular polarization states are discussed subsequently in Sec. 5.1 in context to the results of the corresponding experimental depolarization studies. Although the predicted depolarization trends have been confirmed by experimental studies for certain detection geometries (e.g., forward scattering geometry), one must be careful in generalizing the applicability of these heuristic models, and the resulting predictions/trends, to arbitrary detection geometry/direction. Specifically, the assumption of detection geometry/direction independent depolarization metrics ( $\xi_{l}$ and $\xi_{c}$, see Fig. 2) may not hold for more complex detection geometries than those discussed here. This aspect is also discussed in more detail in Sec. 5.1.

\subsection{Accurate Forward Modeling of Complex Tissue Polarimetry Events}

The approximate analytical approaches described in Sec. 4.1 are mainly aimed at understanding the overall depolarization trends, exploring the dependence of depolarization on the scattering properties of the media, and designing general polarization schemes to discriminate against multiply scattered photons for tissue imaging in "simple" geometries. However, these approaches, while useful, are approximate by their very nature and typically neglect other simultaneously occurring complex tissue polarimetry events (such as linear birefringence, optical activity, etc.). A more encompassing, accurate method is clearly needed for further advances in tissue polarimetry. This can be ac- complished by the polarization-sensitive Monte Carlo (PSMC) techniques. ${ }^{50,60}$

\subsubsection{Tissue polarimetry characteristics}

Before we describe the polarization-sensitive Monte Carlo models, we briefly discuss the various intrinsic tissue polarimetry characteristics that must be dealt with in accurate forward modeling of polarized light-tissue interactions.

Depolarization caused by multiple scattering is the most prominent polarimetry effect in biological tissues. Multiple scattering is caused by the high density of tissue scattering centers, originating from the random fluctuations of the local refractive index in the tissue microstructure (inside the cell and in the extra-cellular matrix). In fact, the tissue scattering centers vary in size (and shape) from micrometer scale and below (sub-cellular structures such as mitochondria, ribosomes, lysosomes, Golgi apparatus, etc.) to several tens of micrometers (whole cells, collagen fibers, etc.). Typical refractive index fluctuations in these scattering structures vary from $\mathrm{n}_{\mathrm{s}} \sim 1.4-1.5$ (the average background refractive index of cytoplasm and interstitial fluid $n_{m}$ 1.34) ${ }^{50}$ Light scattering from all of these microscopic scattering structures contributes in a complex fashion to the observed depolarization of light in tissue. Note that the underlying mechanism of depolarization due to multiple scattering is the scrambling of photon's reference frame (scattering plane) as a consequence of random sequence of scattering events in a variety of scattering directions.

Linear retardance (or birefringence) is the other important tissue polarimetry characteristic. Although not as pervasive as multiple scattering, the anisotropic organized nature of many tissues stemming from their fibrous structure manifests as anisotropic refractive indices parallel and perpendicular to the fibers. Accordingly, these tissues exhibit linear birefringence, which is defined as the difference in refractive indices of the fibers, $\Delta n$ $\left(=n_{e}-n_{o}\right)$, where $n_{e}$ and $n_{o}$ are extraordinary and ordinary refractive indices (the electric field vector or linear polarization of light is perpendicular and parallel to the fiber orientation). This results in phase retardation, also called retardance $[\delta=(2 \pi / \lambda) \Delta n L, L$ is the pathlength] between two orthogonal linear polarization states while propagation through tissue. Extra-cellular matrix proteins (collagen and elastin), actin-myosin fibers, mineralized hydroxyapatite crystals are examples of such birefringent fibers. Various types of tissues, such as muscle, skin, myocardium, bone, teeth, cornea, tendon, cartilage, eye sclera, dura mater, nerve, retina, myelin, etc., possess these birefringent (uniaxial and occasionally biaxial ${ }^{12}$ ) fibrous structures. Typical values of linear birefringence of these biological fibers in the visible wavelength range are in the range $\Delta n$ $=10^{-3}$ to $10^{-2} \cdot{ }^{12}$ Interestingly, even though uniform uniaxial birefringence may not be a direct contributor to depolarization per se, randomly oriented spatial domains of uniform uniaxial birefringent properties may cause polarization loss. ${ }^{11}$ Similarly, circular birefringence (retardance, also called optical rotation in this context) in tissue arises due to the presence of asymmetric optically active chiral molecules like glucose, proteins, and lipids. ${ }^{24}$ Finally, many biological molecules (such as amino acids, proteins, nucleic acids, etc.) also exhibit dichroism or diattenuation effects. The magnitude of diattenuation effects in 


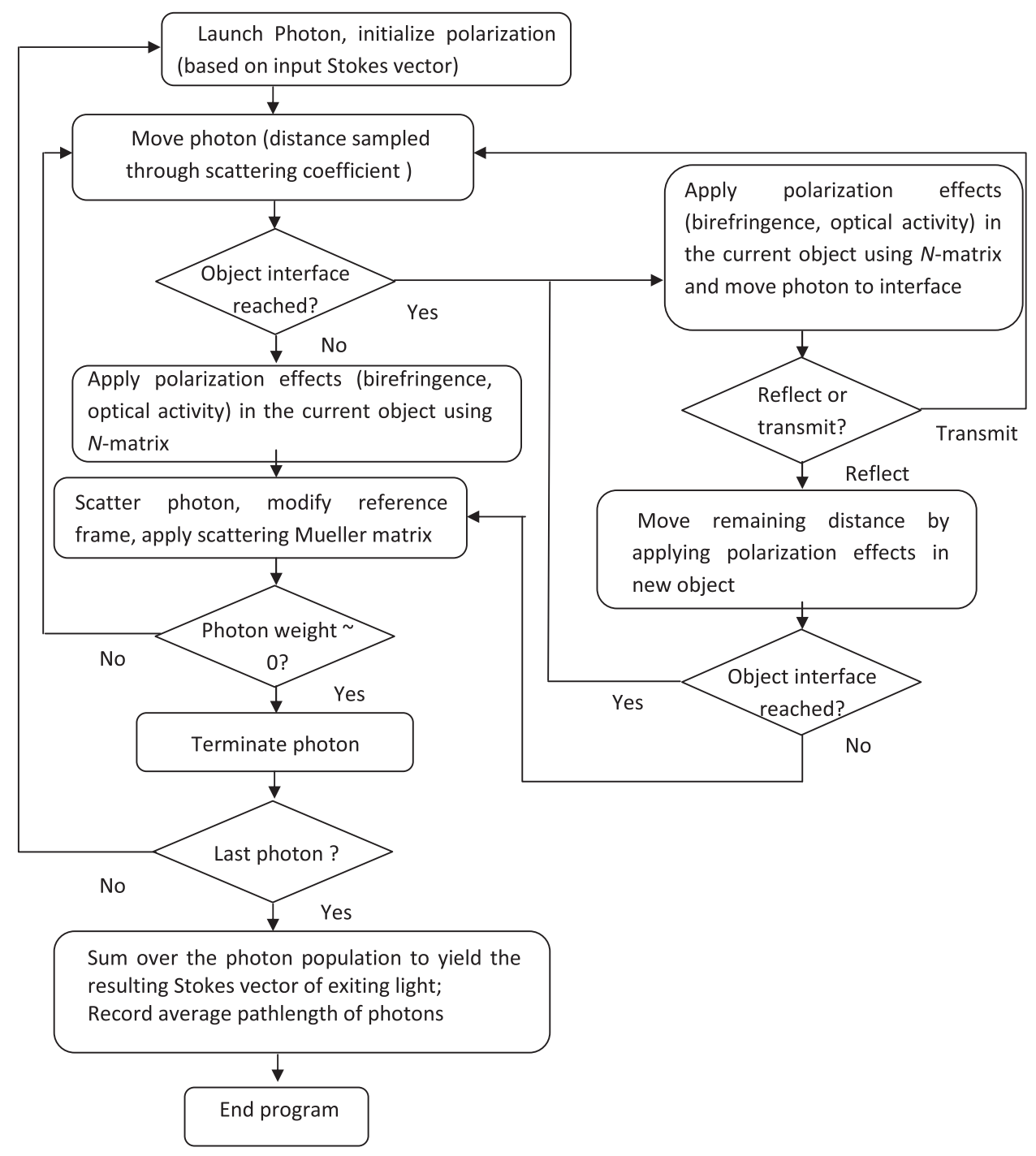

Fig. 3 A flow chart for polarization-sensitive Monte Carlo model. (Adopted from Ref. 15.)

tissue is, however, much lower compared to the other polarization phenomena described above.

\subsubsection{Robust PSMC approach for modeling complex tissue polarimetry characteristics}

The MC technique is a general and robust approach for modeling light transport in random medium..$^{50,60}$ In this statistical approach to radiative transfer, the multiple scattering trajectories of individual photons are determined using a random number generator to predict the probability of each scattering event. The superposition of many photon paths approaches the actual photon distribution in time and space. This approach has the advantage of being applicable to arbitrary geometries and arbitrary optical properties, including ability to simulate heterogeneous media. Most Monte Carlo models were developed for intensity calculations only and neglected polarization information; the most commonly used being the code of Wang et al. ${ }^{60}$ More recently, a number of implementations have incorporated polarization into the Monte Carlo approach. ${ }^{56,61-64}$

A specific example of a polarization-sensitive Monte Carlo model is shown via a flow chart in Fig. $3 .{ }^{15}$ In addition to modifi- cation/incorporation of the position and propagation direction of each photon, polarization information is incorporated by keeping track of the Stokes vectors of propagating photon packets. When the photon encounters a scattering event, a scattering plane and angle are statistically sampled based on the polarization state of the photon and the Mueller matrix of the scatterer. The photon's reference frame is first expressed in the scattering plane and then transformed to the laboratory (experimentally observable) frame through multiplication by appropriate rotation matrices and the Mueller matrix calculated through Mie scattering theory again. For $n$ number of successive scattering events, the resulting Stokes vector $\left(\mathbf{S}_{\mathbf{f}}\right)$ for a photon packet can be expressed as

$$
S_{f}=\left[R^{-1}\left(\phi_{L}\right)\right]\left[M\left(\theta_{n}\right)\right]\left[R\left(\phi_{n}\right)\right] \cdots\left[M\left(\theta_{1}\right)\right] \cdot\left[R\left(\phi_{1}\right)\right] S_{o} .
$$

Here, $S_{O}$ is the input Stokes vector, and $\theta$ and $\phi$ are the scattering and the azimuthal angles respectively. $R\left(\phi_{i}\right)$ is the rotation matrix (connecting the two Stokes vectors that describe the same polarization state with respect to the reference plane and the scattering plane) for the $i$ 'th scattering event 
$(i=1,2 \ldots n)$ given as

$$
[R(\phi)]=\left(\begin{array}{cccc}
1 & 0 & 0 & 0 \\
0 & \cos 2 \phi & \sin 2 \phi & 0 \\
0 & -\sin 2 \phi & \cos 2 \phi & 0 \\
0 & 0 & 0 & 1
\end{array}\right)
$$

$M\left(\theta_{i}\right)$ is the Mie-theory calculated scattering Mueller matrix for the $i$ 'th scattering event (defined in the scattering plane). For isotropic spherical scatterers, symmetry considerations simplify the general form of $M(\theta)$ to yield ${ }^{19}$

$$
M(\theta)=\left[\begin{array}{cccc}
M_{11} & M_{12} & 0 & 0 \\
M_{21} & M_{22} & 0 & 0 \\
0 & 0 & M_{33} & M_{34} \\
0 & 0 & M_{43} & M_{44}
\end{array}\right] .
$$

The symmetry relations for isotropic spherical scatterers allow one to simplify this even further, essentially yielding only four independent matrix elements $\left(M_{11}=M_{22}, M_{12}=M_{21}, M_{33}\right.$ $=M_{44}$ and $\left.M_{43}=-M_{34}\right)$. On the other hand, for scatterers having arbitrary shapes, the form of $M(\theta)$ is far more complex, essentially having nonzero values for all the matrix elements. ${ }^{19}$

Using the aforementioned approach, the evolution of polarization state of each photon packet is tracked following successive scattering events. Absorption effects are handled as in intensity-based Monte Carlo models. ${ }^{50,60}$ Upon encountering an interface (either an internal one, representing tissue domains of different optical properties, or an external one, representing external tissue boundary), the probability of either reflection or transmission is calculated using Fresnel coefficients. As no coherence effects are considered, the final Stokes vector for light exiting the sample in a particular direction is computed as the sum of all the Stokes vectors of appropriate directional photon sub-populations. As previously described, algebraic manipulation of the resulting Stokes vectors for a variety of different polarization inputs can be performed to yield the Mueller matrix of the simulated turbid medium.

In the PSMC approach that handles Stokes vector evolution due to absorption and scattering in a manner just described, other polarimetry effects such as linear birefringence and optical activity can, in principle, be incorporated by including their corresponding Mueller matrices in Eq. (34). However, this is not an obvious modeling step. Difficulty arises in formulating simultaneous polarization effects. Matrix multiplication of the Mueller matrices for individual polarization effects is not commutative $\left(\mathbf{M}_{\mathbf{A}} \mathbf{M}_{\mathbf{B}} \neq \mathbf{M}_{\mathbf{B}} \mathbf{M}_{\mathbf{A}}\right.$, or the Hermitian is nonzero); thus, different orders in which these effects are applied will have different effects on the polarization. Ordered multiplication of these matrices in fact does not make physical sense, as in biological tissue, these effects (such as optical activity due to chiral molecules and linear birefringence due to anisotropic tissue structures) are exhibited simultaneously and not one after the other as sequential multiplication implies. Fortunately, there exists a method to simulate simultaneous polarization effect in clear media through the so-called $N$-matrix formalism, which combines the effects into a single matrix describing them simultaneously. 6,65
The $N$-matrix approach was first developed by Jones, ${ }^{65}$ and a more thorough derivation is provided in Kliger et al. ${ }^{6}$ Briefly, in this approach, the matrix of the sample is represented as an exponential function of a sum of matrices, where each matrix in the sum corresponds to a single optical polarization effect. The issue of ordering of noncommutative matrices is overcome as matrix addition is always commutative, and applies to differential matrices representing the optical property over an infinitely small optical pathlength. These differential matrices are known as $N$-matrices, and their "parent" nondifferential matrices are known as $M$-matrices. The differential $N$-matrices corresponding to each optical property exhibited by the sample are then summed to express the combined effect. The formalism is expressed in terms of $2 \times 2$ Jones matrices applicable to nondepolarizing media, rather than the more commonly used $4 \times 4$ Mueller matrices. For example, the $N$ matrix for combined linear birefringence and optical activity effects is given by ${ }^{61}$

$$
N_{O A+L B}=\frac{1}{2}\left|\begin{array}{l}
i g_{0} \chi \\
-\chi-i g_{0}
\end{array}\right| .
$$

Here, $g_{0}=2 \pi / \lambda \Delta n$ is the phase retardation per unit distance and $\chi$ is the optical rotation per unit distance. The "parent" nondifferential $M$-matrix is calculated from the $N$ matrix to describe the combined effect. ${ }^{61}$ The resulting Jones $M$-matrix is then converted to a Mueller matrix. ${ }^{21}$ Note, however, a Jones matrix description, and thus its conversion to a Mueller matrix, is only valid provided there are no depolarization effects. ${ }^{21}$ This is indeed applicable in the Monte Carlo model, as depolarization is predominantly caused by the multiple scattering, and no depolarization effects should occur between the scattering events. Once converted to a Mueller matrix, this matrix is then applied to the photons as they propagate between scattering events. This approach thus enables the combination of any number of simultaneously occurring polarizing effects. In tissues, linear birefringence and circular birefringence (optical activity) are the important polarimetry characteristics to be added to the multiple scattering effects.

Similar to conventional Monte Carlo modeling, in PSMC also, the scattering and absorption properties are modeled using the optical transport parameters, scattering coefficient $\left(\mu_{s}\right)$ and absorption coefficient $\left(\mu_{a}\right)$. Mie theory is used to compute the single scattering Mueller matrix for known diameter $(D)$ and refractive index of scatterer $\left(n_{s}\right)$ and refractive index of the surrounding medium $\left(n_{m}\right)$. Circular and linear birefringence is modeled through the optical activity $\chi$ in degrees per centimeter, and through the linear anisotropy in refractive indices $\Delta n$ $\left(=n_{e}-n_{o}\right)$, respectively. For simplicity, it is generally assumed that the medium is uniaxial and that the direction of the extraordinary axis and the value for $\Delta n$ is constant throughout the scattering medium ${ }^{61}$ (although our recent research efforts are exploring the effects of multiple uniaxial domains of varying magnitude and orientation of birefringence). Note that as photons propagate between scattering events, the difference in refractive indices $\left[n(\vartheta)-n_{o}\right]$ experienced by them depends on their propagation direction with respect to the extraordinary axis $(\vartheta)$. The effect is modeled using standard formulae describing the angular variation of refractive index in uniaxial medium,

$$
n(\vartheta)=\frac{n_{o} n_{e}}{\sqrt{n_{e}^{2} \cos ^{2} \vartheta+n_{o}^{2} \sin ^{2} \vartheta}} .
$$




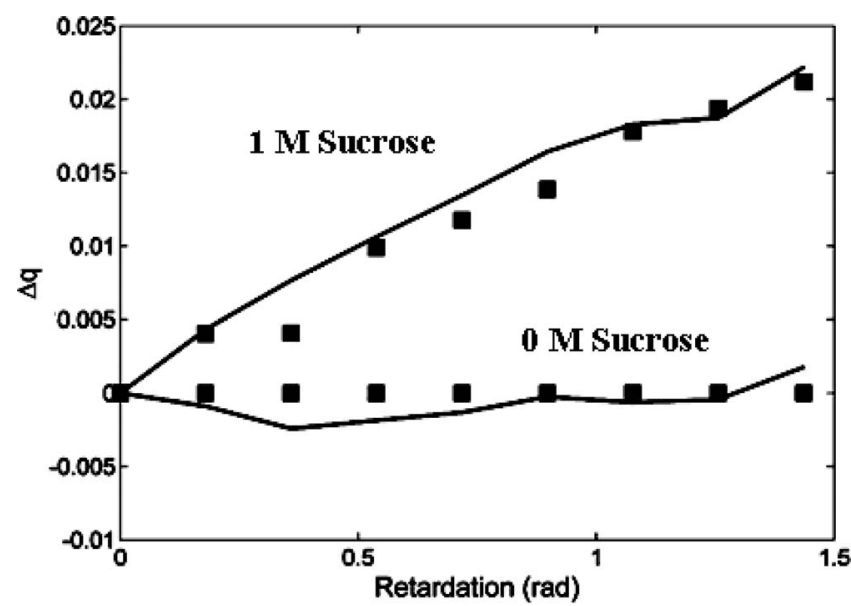

Fig. 4 Experimental measurements (symbols) and Monte Carlo calculations (lines) of the change in the normalized Stokes parameter $q$ with and without optical activity (with and without added sucrose) in the forward $(\gamma=0$ deg) detection geometry with input circularly polarized light and a fixed scattering coefficient of $\mu_{s}=60 \mathrm{~cm}^{-1}$. Linear retardance was varied from $\delta=0$ to $1.4364 \mathrm{rad}$ (corresponding to birefringence variation $\Delta n=0$ to $1.628 \times 10^{-5}$ ) and the magnitude of optical activity was $\chi=1.965 \mathrm{deg} \mathrm{cm}{ }^{-1}$, corresponding to a $1 \mathrm{M}$ sucrose concentration. (Adopted from Refs. 16 and 61.)

In what follows, the ability of this extended (and experimentally validated) polarization-sensitive Monte Carlo model to simulate simultaneous polarization effects in the presence of multiple scattering is illustrated with selected results.

The validity of this model has been tested on experimental tissue simulating phantoms exhibiting simultaneous scattering and polarization properties, which are known and user-controlled a priori. ${ }^{15,16,37}$ These solid optical phantoms were developed using polyacrylamide as a base medium, with sucrose-induced optical activity, polystyrene microspheres-induced scattering, and mechanical stretching to cause linear birefringence. These phantom system mimics the complexity of biological tissues, in that it exhibits simultaneous linear birefringence, optical activity, and depolarization due to multiple scattering. ${ }^{61}$ Figure 4 shows the change in the normalized Stokes parameter $q=Q / I$ with increasing birefringence, measured in phantoms and calculated from the PSMC model in the forward direction of a 1 $\times 1 \times 1 \mathrm{~cm}^{3}$ sample with input circularly polarized light. ${ }^{61}$ The measurements were performed using the experimental system shown in Fig. 1(a). Good agreement between the Monte Carlo model and controlled experimental results is seen. As the input light is transferred from circular to linear polarization due to the increasing sample linear birefringence (the sample in effect acting like a turbid wave-plate), optical rotation due to optical activity of dissolved sucrose is seen as an increase in parameter $q$. No such effect is seen in the absence of chirality.

Figure 5(a) gives an example of the $4 \times 4$ Mueller matrix experimentally recorded in the forward (transmission) detection geometry from a birefringent (extension $=4 \mathrm{~mm}$ for strain applied along the vertical direction, corresponding to a value of linear retardance $\delta=1.345$ rad for a clear phantom of thickness of $1 \mathrm{~cm}$ ), chiral (optical activity $\chi=1.96$ degree $\mathrm{cm}^{-1}$, corresponding to $1 \mathrm{M}$ concentration of sucrose), turbid phantom ( $\mu_{s}$ $=30 \mathrm{~cm}^{-1}$ and $\left.g=0.95\right) .{ }^{37}$ The corresponding matrix gener- (a)

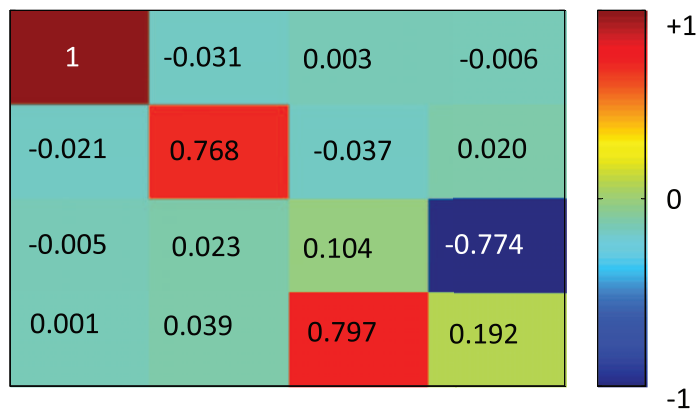

(b)

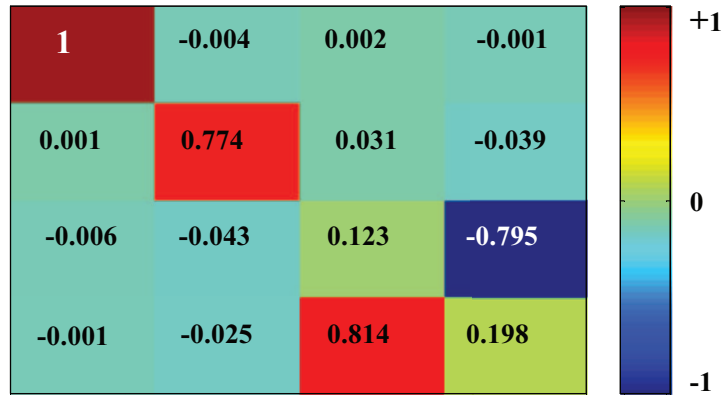

Fig. 5 The $4 \times 4$ Mueller matrices (a) experimentally recorded in the forward (transmission) detection geometry from a birefringent (extension $=4 \mathrm{~mm}$, corresponding to a value of linear retardance $\delta=1.345 \mathrm{rad}$ for a clear phantom), chiral concentration of sucrose $=1 \mathrm{M}, \chi=1.96 \mathrm{deg} / \mathrm{cm})$ turbid $\left(\mu_{s}=30 \mathrm{~cm}^{-1}, g=0.95\right)$ phantom of thickness of $1 \mathrm{~cm}$; (b) generated through the PSMC model, using the same controlled input scattering and polarization parameters (linear birefringence $\Delta n=1.36 \times 10^{-5}$, corresponding to $\delta=1.345 \mathrm{rad}$, $\left.\chi=1.96 \mathrm{deg} \mathrm{cm}^{-1}, \mu_{s}=30 \mathrm{~cm}^{-1}, g=0.95\right)$ as that of the experimental phantom. The results of the decomposition analysis on the experimental Mueller matrix are presented in Fig. 7 and Table 1.

ated through the PSMC model, using the same controlled input scattering and polarization parameters (linear birefringence $\Delta n$ $=1.36 \times 10^{-5}$, corresponding to $\delta=1.345 \mathrm{rad}, \chi=1.96 \mathrm{de}-$ gree $\left.\mathrm{cm}^{-1}, \mu_{s}=30 \mathrm{~cm}^{-1}, g=0.95\right)$ is shown in Fig. 5(b). ${ }^{37}$ In both the experimental and the MC-generated Mueller matrices, the signature of linear retardance is prominent in the matrix elements $M_{34}$ and $M_{43}$ [as is expected for a retarder having an orientation angle $\theta=90 \mathrm{deg}$, see Eq. (10)]. The effect of optical activity is mainly manifest as a difference in $M_{23}$ and $M_{32}$ elements [see Eq. (11)], whereas depolarization effects are most prominently reflected in the diagonal elements of the Mueller matrix. The excellent agreement between the experimental and the simulated Mueller matrices emphasizes the capability of the PSMC model in simulating complex tissue polarimetry effects, including simultaneous optical activity and birefringence in the presence of multiple scattering in any desired detection geometry. However, the complex nature of the recorded Mueller matrix M, with essentially all 16 nonzero elements also underscores a significant problem-how does one extract useful sample metrics from this intertwined array of information? Inverse analysis aimed at quantifying individual polarimetry contributions from "lumped" Mueller matrix are described subsequently (Sec. 6). 
In Sec. 5, we discuss an interesting application of turbid polarimetry for polarization gated imaging of tissue.

\section{Polarized Light Scattering as a Gating Mechanism for Tissue Imaging and Spectroscopy}

Investigation of optical techniques for noninvasive, high resolution imaging of tissue and its underlying structure is an actively pursued area. ${ }^{66,67}$ However, a major difficulty in realizing the potential of the optical techniques for medical imaging is the fact that multiple scattering in tissue leads to loss of directionality that causes severe image blurring. Polarization gating, along with other approaches such as time gating, spatial filtering, and coherence gating have shown some promise in isolating the image bearing photons [the unscattered (ballistic) or weakly scattered (snake) photons] from the image blurring (diffusive or multiply scattered) photons for Ref. 66. Among these attempts, polarization gating has received particular attention because of the relative simplicity of instrumentation, making this a potential tool amenable for clinical use. ${ }^{68}$ The underlying principle of this approach is that multiply scattered light gets depolarized, and therefore by detecting primarily the polarization-retaining component of light scattered from tissue, one can filter out the multiply scattered photons and thus improve imaging resolution. However, for practical implementation of this approach, it is essential to understand the mechanism of depolarization and its dependence on the different morphological parameters of tissue (e.g., the density, size and its distribution, shape and refractive index of the tissue scatterers). A number of experimental studies have therefore investigated these issues. ${ }^{26-30,68-71}$ We shall briefly summarize these studies (with illustrative examples) and discuss their implications for polarimetric tissue imaging.

\subsection{Depolarization of Light in Tissues and Tissue Phantoms: Implications for Polarized Light Tissue Imaging}

Since it is difficult to control and quantify relevant properties in a complex system like tissue, the usual approach followed in most experimental depolarization investigations has been to study the influence of the individual morphological parameters (user controlled and known a priori) in well-characterized tissue simulating phantoms, and relate these to depolarization behavior of light in actual tissue. Suspensions of Intralipid and aqueous suspension of polystyrene (or silica) microspheres have commonly been used to prepare tissue phantoms for these depolarization studies. ${ }^{26-30}$ Usually the size of the scatterers is chosen such that the value of anisotropy parameter $(g)$ is comparable to that of tissue. The concentration of scatterers is then adjusted to yield the value of the scattering coefficient $\mu_{s}$ or the reduced scattering coefficient $\mu_{s}^{\prime}$ desired to mimic the tissue of interest. Molecular dyes of know molar extinction are used to simulate tissue absorption.

For scattering media comprised of Rayleigh scatterers (radius of scatterer $a \ll \lambda$, size parameter $X<2$ and anisotropy parameter $g \leq 0.2$ ), in the forward scattering geometry, depolarization of incident circular polarization is stronger compared to the depolarization of incident linear polarization (as predicted (a)

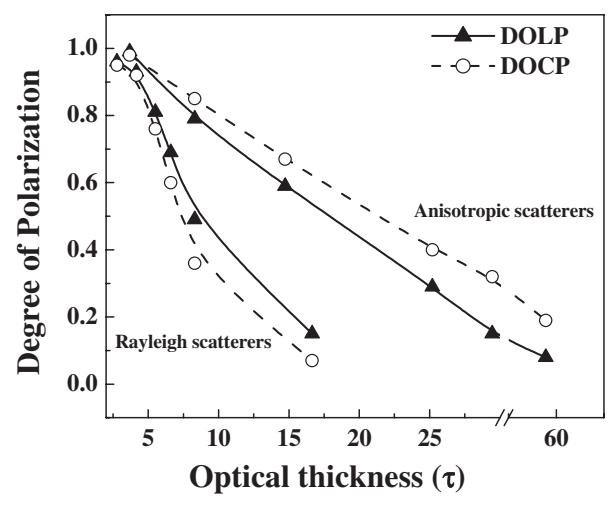

(b)

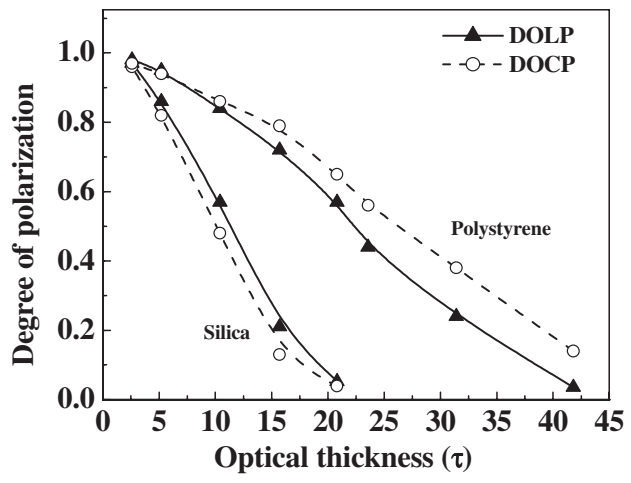

Fig. 6 (a) Measured degrees of linear (DOLP, triangles) and circular (DOCP, circles) polarization as a function of optical thickness $\tau\left(=\mu_{s}\right.$ $\times L, \mu_{s}$ is the scattering coefficient and $L$ is the thickness) from samples prepared using aqueous (refractive index $n_{m}=1.33$ ) suspension of polystyrene microspheres (refractive index $n_{s}=1.59$ ) having mean diameter of $0.11 \mu \mathrm{m}$ (Rayleigh scatterers, $g=0.09, X=0.72$ at $632.8 \mathrm{~nm}$ ) and $1.08 \mu \mathrm{m}$ (Mie scatterers, $g=0.92$ and $X=7.13$ ) as scatterers. The measurements were performed in transmission geometry. The thickness of the sample was $1 \mathrm{~cm}$ and the optical thickness $\tau$ was varied by changing the value of scattering coefficient $\mu_{s}$ through dilution. The symbols are experimental data and the lines are guide for the eye. (Adopted from Ref. 28.) (b) Measured degrees of linear (triangles) and circular polarization (circles) of optical thickness $\tau$ from samples prepared using aqueous suspension of $0.65-\mu \mathrm{m}$ diameter polystyrene $\left(n_{s}=1.59, m=1.03, g=0.86, X=4.29\right)$ and silica microspheres $\left(n_{s}\right.$ $=1.37, m=1.2, \mathrm{~g}=0.89, X=4.29$ ). The measurements were performed in transmission geometry. The thickness of the sample was $1 \mathrm{~cm}$ and the optical thickness $\tau$ was varied by changing the value of scattering coefficient $\mu_{s}$ through dilution. The symbols are experimental data and the lines are guide for the eye. (Adopted from Ref. 29.)

by heuristic models described in Sec. 4.1). The reverse is the case for scattering media comprised of large sized Mie scatterers ( $a \geq \lambda, X>2, g \geq 0.7$ ). Moreover, the overall strength of depolarization is considerably weaker in the latter case. Typical results for such experimental depolarization measurements are shown in Fig. 6(a), where the degree of polarization for transmitted light is shown as a function of optical thickness $\tau$ ( $=\mu_{s} \times L, \mu_{s}$ is the scattering coefficient and $L$ is the physical thickness) from samples prepared using aqueous (refractive index $n_{m}=1.33$ ) suspensions of polystyrene microspheres (refractive index $n_{s}=1.59$ ) having mean diameter of $0.11 \mu \mathrm{m}$ (Rayleigh scatterers, $g=0.09, X=0.72$ at $632.8 \mathrm{~nm}$ ) and 
$1.08 \mu \mathrm{m}$ (Mie scatterers, $g=0.92$ and $X=7.13$ ). ${ }^{28,30}$ Again, these results are in qualitative agreement with the predictions of the corresponding theoretical models for polarized light transport in turbid medium (described in Sec. 4.1). The weaker depolarization for media comprised of large sized scatterers can be explained by noting that with increasing value of size parameter of scatterer $X$, forward scattering becomes more predominant (value of $g$ increases), resulting in weaker randomization of photon's propagation direction when forward (transmission) detection geometry is employed. ${ }^{53-56,68}$ The difference in relative rate of depolarization of linearly and circularly polarized light can be attributed to the different mechanism of depolarization of the two. While randomization of the incident field vector's direction as a consequence of multiple scattering events is solely responsible for the depolarization of incident linear polarization state, the depolarization of circular polarization state is additionally influenced by the randomization of the helicity (handedness). ${ }^{54}$ Note that scattering at large angles flips the helicity of the circular polarization state. For Rayleigh scatterers, the critical scattering angle, above which the helicity of circularly polarized photons is reversed, is $90 \mathrm{deg}$. However, this helicity flip angle increases (>90 deg) with increasing size of the scatterers. ${ }^{28,68}$ In a turbid medium, light travels along many possible zig-zag paths, having contributions from scattering at various angles. For Rayleigh scatterers (where forward and back scattering is $\sim$ equally likely), the contribution of the large angle scattered photons is greater as compared to the larger sized Mie scatterers (where forward scattering predominates), ensuing stronger randomization of helicity and thus resulting in stronger depolarization of circularly polarized light in Rayleigh media $(X<2$, $g \leq 0.2$ ). As the scatterer size increases, the additional cause of depolarization of circularly polarized light, the flipping of helicity also gets considerably reduced (due to a relatively lesser contribution of large angle scattered photons coupled with the fact that the helicity flip angle is also larger), resulting in weaker depolarization of circularly polarized light for anisotropic $(g \geq 0.7)$ scattering media comprised of Mie scatterers $(X>2)$. It may also be pertinent to note that the corresponding theoretical variation of the length scales of depolarization $\left(\xi_{l}\right.$ and $\xi_{c}$ ) showed a general decreasing trend beyond a size parameter value of $X \geq 10$ (see Fig. 2, Sec. 4.1). This anomalous depolarization behavior has been attributed to the Mie resonance effects present in the large sized scatterers. ${ }^{53}$

The depolarization is also influenced by the refractive index contrast present in the turbid medium. ${ }^{28,55}$ Depolarization characteristics of Mie-like anisotropic scattering media $(g \geq 0.7)$ comprised of low refractive index scatterers [e.g., silica in water, low value of the relative refractive index $m\left(=n_{s} / n_{m}\right) \sim$ 1.03 to 1.05 ] is distinctly different from those comprised of high refractive index scatterers $(m \gg 1.05$, e.g., polystyrene in water). In fact, the depolarization behavior in low-indexcontrast Mie medium surprisingly resembles the behavior for Rayleigh scatterers. ${ }^{28}$ This is illustrated in Fig. 6(b), where the measured degrees of linear and circular polarization from samples prepared using aqueous suspension of $0.65-\mu \mathrm{m}$ diameter polystyrene $\left(n_{s}=1.59, m=1.2, g=0.86, X=4.29\right)$ and silica microspheres $\left(n_{s}=1.37, m=1.03, g=0.89, X=4.29\right)$ are displayed. ${ }^{29}$ Despite having a slightly larger value for $g$, depolarization of both linearly and circularly polarized light is stronger for the samples having silica microspheres as compared to those having polystyrene microspheres as scatterers. Moreover, for the silica microspheres samples, circular polarization actually decays more rapidly as compared to linear polarization, opposite of the Mie trends previously described and in fact more associated with Rayleigh scatterers exhibiting small $g(\leq 0.2)$. This interesting behavior originates from the fact that the anisotropic scatterers $(g \geq 0.7, X \gg 2)$ having a lower value of relative refractive index $m[m \leq 1.05,(m-1) \mathrm{X} \ll 1]$ can be treated to be in the weak scattering regime, where each volume element inside the scatterer can be assumed to be giving electrical dipole scattering in an independent manner. ${ }^{29,56}$ The corresponding scattering matrix becomes similar in nature to Rayleigh scatterers (ideal dipole scattering). The retention of this dipolar behavior thus leads to depolarization characteristics of such weakly fluctuating anisotropic scattering medium much similar to that of Rayleigh scatterers. Since biological tissue can be considered as a random continuum of inhomogenities having weak fluctuations in refractive indices (refractive indices of scattering inhomogenity $n_{s} \sim 1.37$ to 1.41 , of background medium $n_{m} \sim 1.33$ to 1.35 , thus $m \leq 1.05$ ), its depolarization behavior is thus expected to be similar to that of Rayleigh scatterers. ${ }^{56}$ This indeed has been observed in experimental depolarization studies on actual tissues, ${ }^{26,27}$ despite the potentially mistaken paradigm that "circular is better in Mie-scattering."

Once again, caution must be exercised in generalizing the depolarization behavior in a turbid medium based on the experimental results described above. As noted previously, the quantitative rate of depolarization and the relative depolarization behavior of linearly and circularly polarized light may also be additionally influenced by detection direction/geometry. In order to illustrate this point, let us consider the following: incident circularly polarized photons getting multiply scattered in an anisotropic $(g \geq 0.7)$ scattering medium, with a photodetector positioned for measurements in the backward direction. At detection points sufficiently close to the point of illumination, a major component of the detected photons are the helicity flipped backscattered photons. In contrast, as one moves away from the point of illumination, there would be significant contribution of the sub-population of helicity preserving photons which have undergone a series of forward scattering events to emerge through the backward direction. The observed depolarization at a chosen detection position in the backward direction will thus be the resultant of a complex combination of these many possible zig-zag photon paths, which may differ from the experimental trends presented above.

Finally, the efficiency of the polarization gating scheme for filtering out the multiply scattered light is determined by the degree to which incident polarized light becomes randomized or depolarized. It is thus desirable that the multiply scattered photons (whose direction information has been randomized) should also be depolarized to a greater extent. These photons can then be efficiently filtered out by imposing a polarization gate that will preferentially detect the polarization-retaining component of light emerging from the medium. Based on the results of the depolarization studies discussed above, the following inferences can be made: i. For scattering media comprised of Rayleigh scatterers, polarization (specifically circular polarization) can be used as an effective scheme to filter out multiply scattered photons. ii. For anisotropic scattering media comprised of high refractive index, large-sized Mie scatterers, the 
polarization filtering schemes will be less efficient. iii. As in the case of Rayleigh scattering, for anisotropic scattering media comprised of low refractive index, large-sized scatterers (or weakly fluctuating random media), polarization gating should be an efficient method for discriminating ballistic and snake from diffusive photons. Since in terms of the scattering properties, biological tissue falls in category iii., polarization-gated methods may be advantageous compared to corresponding polarizationblind methods for tissue imaging. Several research groups have therefore actively pursued various polarization gating schemes and strategies for imaging of tissue and its underlying structures, as summarized below.

\subsection{Polarization Gated Imaging and Spectroscopy of Tissue}

The simplest form of polarized light imaging method employs illumination of tissue by either linearly or circularly polarized light, and recording of images with either the same orientation of the polarizer and the analyzer (co-polarized, $I_{\mathrm{co}}$ ) or with the opposite orientation (cross-polarized, $I_{\text {cross }}$ ). A similar approach for linear polarization illumination has already become common practice in dermatology. ${ }^{72}$ Viewing the skin with co(or crossed-) polarization channels allows the dermatologist to either observe the glare from the air/skin surface and surface details (or to suppress it for a better view the deeper subsurface tissue structures). Jacques et al. ${ }^{17,73,74}$ modified this scheme further for high resolution imaging of the texture of the superficial (sub-surface) skin structures. In this approach, the glare from the air/glass/skin interfaces was removed through optical coupling (using index matching liquid) of a glass plate in contact with the skin and by off-normal illumination. The multiply scattered photons were filtered out by extracting the polarization preserving component of the scattered light employing either the polarization difference scheme $\left(I_{\mathrm{co}}-I_{\mathrm{cross}}\right)$ or the degree of polarization scheme $\left[\mathrm{DOP}=\left(I_{\mathrm{co}}-I_{\text {cross }}\right) /\left(I_{\mathrm{co}}+I_{\text {cross }}\right)\right]$. In fact, the DOP imaging has subtle advantages over the polarization difference imaging in that the DOP imaging is relatively insensitive to spatial variation of illuminating light and variations in pigmentation. This follows because the DOP image is formed by the ratio of the numerator (primarily comprised of superficial subsurface reflectance) and the denominator (representative of total subsurface reflectance). Based on this simple principle, a polarized light camera system has been developed and used in clinical studies for finding skin cancer margins. ${ }^{17}$

Morgan et al. ${ }^{75}$ developed another elegant polarization scheme for eliminating surface reflection in backscattering imaging of superficial tissue layers, obviating the use of index optically flat plates (and index matching fluid) and angled illumination (thus enabling co-axial detection). It was shown that a suitable combination of co- and cross-polarized images acquired using linear and circular polarization illumination can be used to simultaneously filter out both the multiply scattered light and the surface reflection. The relative efficacies of the different polarization gating schemes for tissue imaging have also been investigated in detail by this group. ${ }^{69}$

Demos et al. $^{76}$ have developed a methodology based on both spectral and polarization discrimination of backscattered photons for deep subsurface imaging of tissue. This approach, known as spectral polarization difference imaging, exploits the difference in light penetration of different wavelengths in combination with polarization filtering for selectively imaging different tissue depths.

Orthogonal polarization spectral imaging is another important development in polarimetric tissue imaging. ${ }^{77}$ In this approach, the tissue is illuminated with linearly polarized light and the backscattered light is detected in cross-polarization channel. Since the detected depolarized scattered light (passing through orthogonal polarizer), penetrates deeper in tissue, it effectively back-illuminates the absorbing material (e.g., blood present in tissue) in the foreground. Appropriate choice of illuminating wavelengths (typically in the range of $550 \mathrm{~nm}$, optimized based on the absorption of the hemoglobins present in blood) enables one to image blood vessels in tissue. This approach has therefore been exploited for quantitative assessment of the blood vessels in microcirculation for a number of diagnostic applications. ${ }^{78}$

Apart from the few aforementioned examples, several other experimental approaches such as rotating polarizer imaging, ${ }^{79}$ complete $4 \times 4$ (16 element) Mueller matrix imaging, ${ }^{80}$ and others have also been explored for polarimetric tissue imaging.

Investigation of the optical spectroscopic techniques (such as fluorescence, Raman, and elastic scattering spectroscopy) for biomedical diagnosis is an area of considerable current interest, as optical methods can facilitate noninvasive quantitative diagnosis and can provide (functional/biochemical/compositional/metabolic) information beyond tissue structure. Indeed, very encouraging results have already been obtained in using these approaches for early detection of cancer. ${ }^{81,82}$ In this context, polarization spectroscopy can offer several important advantages over the conventional polarization-blind measurements. Specifically, the polarization gating schemes can be exploited for depth selective spectroscopic measurements in tissue, which can improve the diagnostic efficacy. ${ }^{83,84}$ The underlying principle for polarization gating as a depth selective technique is as described before-the photons which are scattered (or re-emitted) from deeper tissue layers undergo multiple scattering events and are depolarized to a larger extent. Polarization gating thus effectively selects photons which have not traveled beyond a few scattering mean free paths (mpf $\sim 100 \mu \mathrm{m}$ in tissue). Polarization resolved spectroscopic approaches are thus expected to be particularly suitable for early detection of epithelial cancers (where the majority of human malignancies originate). Indeed, polarized elastic backscattering spectroscopy has been successfully exploited for quantitative measurement of epithelial cellular structures as signature of precancerous changes in human tissues. ${ }^{83,84}$ The method comprises of excitation of tissue by polarized white light and detection of the polarization-retaining component of the backscattered light. The detected signal (primarily comprising of singly scattered photons from superficial epithelial layer) exhibited a fine structure component periodic in wavelength, thought to be primarily indicative of Mie scattering by surface epithelial cell nuclei. ${ }^{10,83}$ By analyzing the amplitude and frequency of this signal using Mie theory, the size distribution and the refractive index of the nuclei were extracted, and related to pathological status of the examined tissues. Further, the inverse power law spectral dependence of the polarization-gated elastic scattering signal was related to the self-similar (fractal) nature of microscale fluctuations of local refractive index in tissue. ${ }^{84}$ Tissue self-affinity was successfully quantified using fractal-Born approximation-based 
light scattering models, yielding diagnostically important microoptical parameters of tissue, namely, the fractal-Born spectral component, the fractal dimension, and the fractal upper scale. The significant differences observed in the estimated size (and distribution), refractive index of epithelial cell nuclei, and the fractal micro-optical parameters of the normal and dysplastic (epithelial precancer) tissues indicate that these may serve as potential biomarkers for precancer.

In continuing development on polarization-resolved elastic backscattering spectroscopy, Backman et al. developed an advanced light scattering instrument called "four-dimensional elastic light scattering fingerprinting” (Refs. 85-87). This approach facilitates acquisition of light scattering data in several dimensions, namely i. wavelength of light $(\lambda)$, ii. the scattering polar angle $(\theta)$, iii. the azimuthal angle $(\phi)$ of scattering, and iv. polarization of scattered light. Briefly, in this approach, tissue is excited by collimated linearly polarized white light, and the backscattered light collected by a Fourier lens is transmitted through an analyzing polarizer and is relayed to an imaging spectrometer coupled with a CCD for photodetection. The Fourier lens projects the angular (polar) distribution of scattered light onto the entrance slit of the spectrometer. The spectrometer disperses the scattered light according to its spectral content in a direction orthogonal to the slit and projects it into the CCD. Thus the instrument records a matrix of the distribution of scattered light intensity for various wavelengths $(\lambda)$ and angles of scattering $(\theta)$. The azimuthal angle $(\phi)$ of scattering is further selected by rotating the polarizer in the delivery arm of the system and the analyzer allows for detection of co-polarized $\left(I_{\mathrm{co}}\right)$ and crossedpolarized $\left(I_{\text {cross }}\right)$ component of the detected light. Importantly, recording of differential polarization signal $\left[\Delta I(\lambda, \theta)=I_{\text {co }}(\lambda, \theta)\right.$ - $\left.I_{\text {cross }}(\lambda, \theta)\right]$ allows one to capture elastic scattering signature from superficial tissue structures (located within the first 50 to $100 \mu \mathrm{m}$ of tissue surface which typically includes the epithelial cell layer). The simultaneous recording and analysis of spectral, angular and azimuthal variation of scattered light has important advantages for early diagnosis of cancers and precancers. ${ }^{85,86}$ Indeed, use of inverse Mie theory and Fractal analysis on the recorded wavelength and angular variation of scattering signal respectively, have been shown to yield wealth of information on micro-and nanoarchitectural changes in intraepithelial structures as signatures of precancerous alterations. ${ }^{85}$ The same group has also recently developed an endoscopic fiber-optic probe for in vivo polarization-gated (depth-selective) elastic scattering spectroscopic measurements and have initially explored the technique for quantification of microvascular blood supply changes associated with neoplasia. ${ }^{88}$ Georgakoudi et al. also recently developed similar strategy based on polarization gating for depth resolved light scattering spectroscopic measurements from tissue. $^{89}$

Polarization gated fluorescence spectroscopic measurements have also shown early promise to potentially impact some of the unresolved issues in fluorescence-based diagnosis of cancer. Fluorescence signal from layered epithelial tissues, detected with conventional measurement technique, is due to contributions from different endogenous fluorophores (having different quantum yields, lifetimes, and overlapping spectral line shapes) present in the superficial epithelial layer and the underlying connective tissue (stroma). Depth-resolved fluorescence measurement should turn out to be advantageous for early detection of precancer, because the fluorescence contrast from cancerous and noncancerous sites depends on the differences in depth distribution of the respective fluorophores. Polarization resolved fluorescence measurements have been successfully exploited to decouple, isolate, and quantify fluorescence contributions from tissue layers, which in turn helps improve the diagnostic efficacy of the technique. ${ }^{90-92}$

In summary, polarization gating is playing important roles toward realization of the optical techniques for improved resolution imaging of tissue with enhanced depth selectivity. Moreover, combination of polarization gating with spectroscopic (elastic scattering, fluorescence, and Raman ${ }^{93}$ ) measurements offers important advantages over corresponding polarization blind methods for disease diagnosis, as briefly outlined and illustrated above. For a more comprehensive account, the reader is referred to Refs. 83-93. We now turn to the challenging problems of analysis/interpretation of individual intrinsic tissue polarimetry parameters, in the context of their applications in diagnostic photomedicine.

\section{Tissue Characterization via Polarimetry: Analysis/Interpretation of the Intrinsic Tissue Polarization Properties}

As noted previously, the most common intrinsic tissue polarimetry effects, in rough order of prevalence/importance are depolarization, linear birefringence, and optical activity. Each of these, if separately extracted, holds promise as a useful biological metric. For example, chirality-induced optical rotation can be linked to the glucose concentration in the medium; ${ }^{16,24}$ changes in tissue mechanical anisotropy (resulting from disease progression or treatment response) can be probed by birefringence measurements. ${ }^{12,15}$ Despite the wealth of the interesting properties that can be probed with tissue polarimetry, numerous complexities due to multiple scattering and due to the simultaneous occurrence of these polarization effects present formidable challenges, both in terms of accurate measurements and in the extraction/unique interpretation of the constituent polarization parameters. Multiple scattering not only causes extensive depolarization, it also alters the polarization state of the residual polarization preserving signal in a complex fashion, for example by scattering-induced diattenuation and by scattering-induced changes in the orientation of the polarization vector. ${ }^{14,31}$ Moreover, simultaneous occurrences of several polarization effects contribute in a complex inter-related way to the measurable Stokes vectors or Mueller matrix elements. These therefore represent several lumped effects with much "interelement cross talk," masking potentially interesting polarization biometrics and hindering their interpretation. Methods to account for the effects of multiple scattering, and to decouple the individual contributions of several effects occurring simultaneously, are thus needed.

Earlier attempts for tissue polarimetry analysis methods have mainly employed semi-empirical formulations by selectively picking out appropriate Stokes vector or Mueller matrix elements (or a combination of elements), for isolating and analyzing the polarization signatures arising from different tissue anatomical or compositional characteristics. ${ }^{94}$ Recent studies have explored more general polarimetry analysis models for 
extraction, quantification, and interpretation of the intrinsic tissue polarimetry characteristics. ${ }^{37,38}$ Specifically, measurement of complete (16) Mueller matrix elements and their analysis through different decomposition methods have been explored for this purpose, as summarized below.

\subsection{Overview of the Inverse Polarimetry Analysis Methods Based on Mueller Matrix Decomposition}

The Mueller matrix decomposition approach aims to solve the complicated inverse problem, that is, to extract constituent polarization properties from a given lumped system Mueller matrix of any unknown complex system. This method consists of representing a given Mueller matrix $\mathbf{M}$ as an equivalent combination of basis matrices, each of which describes an individual polarimetry effect. The decompositions can be broadly classified into two categories i. the product decomposition and ii. the sum decomposition. Among these, the product decompositions have seen more extensive use in tissue polarimetry investigations, and are thus discussed in detail here.

The product decomposition consists of representing $\mathbf{M}$ as a product of three basis matrices ${ }^{23}$

$$
\mathbf{M} \Leftarrow \mathbf{M}_{\Delta} \cdot \mathbf{M}_{\mathbf{R}} \cdot \mathbf{M}_{\mathbf{D}},
$$

with the $\Leftarrow$ symbol used to signify the decomposition process. Here, the matrix $\mathbf{M}_{\Delta}$ describes the depolarizing effects of the medium, $\mathbf{M}_{\mathrm{R}}$ accounts for the effects of linear birefringence and optical activity, and $\mathbf{M}_{\mathrm{D}}$ includes the effects of linear and circular diattenuation.

Once calculated, these constituent basis matrices can be further analyzed to derive quantitative individual polarization medium properties, as summarized below. ${ }^{23}$ Starting on the right-hand side of Eq. (39), the diattenuation matrix $\mathbf{M}_{\mathbf{D}}$ is defined as

$$
\mathbf{M}_{\mathbf{D}}=\left[\begin{array}{ll}
1 & \vec{d}^{T} \\
\vec{d} & m_{D}
\end{array}\right]
$$

where $m_{D}$ is a $3 \times 3$ sub-matrix, the standard form of which is

$$
m_{D}=\sqrt{\left(1-d^{2}\right)} I+\left(1-\sqrt{\left(1-d^{2}\right)}\right) d^{\wedge} d^{\wedge T},
$$

where $I$ is the $3 \times 3$ identity matrix, $\vec{d}$ is diattenuation vector, and $d^{\wedge}$ is its unit vector, defined as

$$
\vec{d}=\frac{1}{M(1,1)}[M(1,2) M(1,3) M(1,4)]^{T} \text { and } d^{\wedge}=\frac{\vec{d}}{|\vec{d}|} .
$$

The magnitude of diattenuation $|\vec{d}|$, a potentially useful intrinsic bio-polarimetry metric, can be determined from

$$
d=\frac{1}{M(1,1)} \sqrt{M(1,2)^{2}+M(1,3)^{2}+M(1,4)^{2}} .
$$

The remaining product of the retardance and the depolarizing matrices follows from Eq. (39) as

$$
\mathbf{M}_{\Delta} \mathbf{M}_{\mathbf{R}}=\mathbf{M}^{\prime}=\mathbf{M} \mathbf{M}_{\mathbf{D}}^{-1} .
$$

The matrices $\mathbf{M}_{\Delta}, \mathbf{M}_{\mathbf{R}}$, and $\mathbf{M}$ have the following form

$$
\begin{aligned}
\mathbf{M}_{\Delta} & =\left[\begin{array}{ll}
1 & \overrightarrow{0}^{T} \\
P_{\Delta} & m_{\delta}
\end{array}\right] ; \quad \mathbf{M}_{\mathbf{R}}=\left[\begin{array}{cc}
1 & \overrightarrow{0}^{T} \\
\overrightarrow{0} & m_{R}
\end{array}\right] ; \quad \text { and } \\
\mathbf{M}^{\prime} & =\left[\begin{array}{ll}
1 & \overrightarrow{0}^{T} \\
P_{\Delta} & m^{\prime}
\end{array}\right] .
\end{aligned}
$$

Here $P_{\Delta}=(\vec{P}-m \vec{d}) /\left(1-d^{2}\right)$, the polarizance vector $\vec{P}$ $=\frac{1}{M(1,1)}[M(2,1) M(3,1) M(4,1)]^{T}\left[m_{\Delta}\right.$ and $m_{R}$ are $3 \times 3$ submatrices of $\mathbf{M}_{\Delta}$ and $\mathbf{M}_{\mathbf{R}}$ ]. Similarly, $m^{\prime}$ is a $3 \times 3$ sub-matrix of $\mathbf{M}$

$$
m^{\prime}=m_{\Delta} m_{R}
$$

The sub-matrix $m_{\Delta}$ can be computed by solving the eigenvalues for the matrix $m m^{T}{ }^{23}$ This can then be used to construct the depolarization matrix $\mathbf{M}_{\Delta}$. From the elements of $\mathbf{M}_{\Delta}$, net depolarization coefficient $\Delta$, another potentially useful biometric, can be calculated using Eq. (9).

The remaining retardance $\mathbf{M}_{\mathbf{R}}$ matrix can be computed from Eqs. (44) and (45). The value for total retardance ( $R$ is a parameter that represents the combined effect of linear and circular birefringence) can be determined from $\mathbf{M}_{\mathbf{R}}$ :

$$
R=\cos ^{-1}\left\{\frac{\operatorname{Tr}\left(M_{R}\right)}{2}-1\right\} \text {. }
$$

$\mathbf{M}_{\mathbf{R}}$ can be further expressed as a combination of a matrix for a linear retarder (having a magnitude of linear retardance $\delta$, its retardance axis orientation angle $\theta$ ) and a circular retarder (optical rotation with magnitude of $\psi$ ). Using the standard forms of the linear retardance and optical rotation matrices [Eqs. (10) and $(11)]$, the values for optical rotation $(\psi)$ and linear retardance $(\delta)$ can be determined from the elements of the matrix $\mathbf{M}_{\mathbf{R}}$ as ${ }^{37}$

$$
\begin{aligned}
\delta= & \cos ^{-1} \\
& \times\left\{\sqrt{\left[\mathbf{M}_{\mathbf{R}}(2,2)+\mathbf{M}_{\mathbf{R}}(3,3)\right]^{2}+\left[\mathbf{M}_{\mathbf{R}}(3,2)-\mathbf{M}_{\mathbf{R}}(2,3)\right]^{2}}-1\right\}
\end{aligned}
$$

and

$$
\psi=\tan ^{-1}\left[\frac{\mathbf{M}_{\mathbf{R}}(3,2)-\mathbf{M}_{\mathbf{R}}(2,3)}{\mathbf{M}_{\mathbf{R}}(2,2)+\mathbf{M}_{\mathbf{R}}(3,3)}\right] .
$$

To recap, the above decomposition process [Eqs. (39)-(49)] operates on an arbitrary sample matrix $\mathbf{M}$, and yields several medium-specific intrinsic polarimetry metrics with promising tissue diagnostic values, namely diattenuation $d$, depolarization $\Delta$ (linear, circular and total), linear retardance $\delta$ (and its orientation $\theta$ ), and optical rotation $\psi$.

The product decomposition process described above was originally proposed by $\mathrm{Lu}$ and Chipman, and is accordingly known as Lu-Chipman decomposition. ${ }^{23}$ Note that the multiplication order in Eq. (39) is ambiguous (due to the noncommuting nature of matrix multiplication, $\mathbf{M}_{\mathbf{A}} \mathbf{M}_{\mathbf{B}} \neq \mathbf{M}_{\mathbf{B}} \mathbf{M}_{\mathbf{A}}$ ), so that a total of six different decompositions (orders of multiplication) are possible. These six different decompositions are classified into two families, depending upon the location of the matrices for the depolarizer and the diattenuator. ${ }^{95}$ The three decompositions with the depolarizer located after the diattenuator form the first family [to which Eq. (39) belongs]. The three decomposition orders with the depolarizer set before the diattenuator 
constitute the other family: ${ }^{95}$

$$
\left(\mathrm{M}_{\Delta \mathrm{D}}\right. \text { family) }
$$

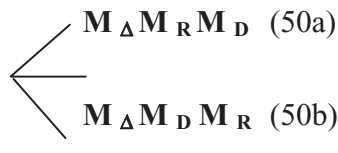

$\mathbf{M}_{\mathbf{R}} \mathbf{M}_{\Delta} \mathbf{M}_{\mathbf{D}}(50 \mathrm{c})$
( $\mathrm{M}_{\mathrm{D} \Delta}$ family)

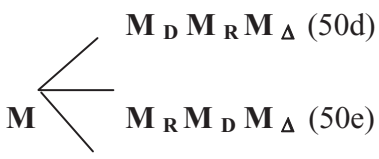

$\mathbf{M}_{\mathbf{D}} \mathbf{M}_{\Delta} \mathbf{M}_{\mathbf{R}}(50 \mathrm{f})$

Algorithms for decomposing with multiplication order of the basis matrices reverse to that of the $\mathrm{Lu}-\mathrm{Chipman}$ decomposition has also been developed $\left[\mathbf{M}=\mathbf{M}_{\mathbf{D}} \cdot \mathbf{M}_{\mathbf{R}} \cdot \mathbf{M}_{\Delta}\right.$, known as the reverse decomposition, Eqs. (50d) above]. It has been shown that the other possible decompositions can be obtained from these two decompositions by using similarity transformations. ${ }^{95}$ In a recent study, ${ }^{96}$ we explored the six possible orders in terms of their physical realizability and their ability to correctly derive the input values of the constituent characteristics $d, \Delta, \delta, \theta$, and $\psi$. It was concluded that in tissue polarimetry (in the absence of large $d$-values), the Lu-Chipman order and its reverse are the correct descriptors of the medium. Reassuringly, when using these two decomposition orders, the only difference in the derived $d, \Delta$, $\delta, \theta$, and $\psi$ values was occasional sign change (e.g., $\psi \leftrightarrow-\psi$ ), thus capturing the important magnitudes of all the polarization metrics in a self-consistent manner.

In other attempts to lift any remaining ambiguity associated with the ordering of the basis matrices, a more general kind of product decomposition, namely, the symmetric decomposition has also been developed recently. ${ }^{97}$ This decomposition factorizes an arbitrary depolarizing Mueller matrix $\mathbf{M}$ down to a diagonal depolarizer $\mathbf{M}_{\Delta}$ placed "in the middle" (i.e., not in front or at the back) of an equivalent optical sequence containing diattenuators and retarders $\left(\mathbf{M}=\mathbf{M}_{\mathbf{D} 2} \cdot \mathbf{M}_{\mathbf{R} 2} \cdot \mathbf{M}_{\Delta} \cdot \mathbf{M}_{\mathbf{R} 1}{ }^{\mathbf{T}} \cdot \mathbf{M}_{\mathbf{D} 1}\right)$.

As noted earlier, in addition to the product decompositions, there exists another approach of the sum decomposition (known as the Cloude decomposition). ${ }^{98}$ In this process, an arbitrary depolarizing Mueller matrix $\mathbf{M}$ is represented as a sum of a nondepolarizing Mueller matrix $\left(\mathbf{M}_{\mathbf{n} \Delta}\right)$ and an ideal depolarizer $\left(\mathbf{M}_{\Delta}\right)$. The nondepolarizing components can further be factorized into products of basis Mueller matrices of diattenuators and retarders, by using the previously mentioned product decomposition. However, this type of decomposition has not been used in tissue polarimetry.

Note that implementation of the decomposition approaches for polarized light examination of complex systems like tissue requires comprehensive validation. Specifically, one needs to know how the decomposition-derived polarization parameters are influenced by the choice of the decomposition process, ordering of the basis matrices, propagation path of multiply scattered photons, detection geometry, and in fact whether the values of the derived polarization parameters represent the true polarimetric medium properties. These issues have therefore been investigated in detail by us and others. ${ }^{15,37,99}$ In what follows, we illustrate the validity of the decomposition formalisms with selected results from tissue-simulating phantoms of increasing biological complexity.

\subsection{Selected Results and Trends Illustrating the Validity of the Mueller Matrix Decomposition Method}

Figure 7 gives an example of the decomposition of the $4 \times 4$ Mueller matrix experimentally recorded in the forward detection geometry from a solid polyacrylamide phantom exhibiting simultaneous linear birefringence, optical activity, and depolarization effect [the same phantom whose recorded Mueller matrix was presented in Fig. 5(a)]. ${ }^{37}$ The decomposed depolarization $\left(\mathbf{M}_{\Delta}\right)$, retardance $\left(\mathbf{M}_{\mathbf{R}}\right)$, and diattenuation $\left(\mathbf{M}_{\mathbf{D}}\right)$ matrices are also shown in figure 7 . In contrast to the complex nature of the recorded Mueller matrix (essentially having all 16 nonzero elements), the derived basis matrices exhibit simpler features with many of the off-diagonal elements being zero. These are thus directly amenable for further quantification. The individual polarization parameters $(\Delta, d, \delta$, and $\psi)$ were retrieved from the decomposed basis matrices [using Eqs. (9), (43), (48), and (49)]. The determined values for these parameters are listed in Table 1, showing excellent agreement with the controlled inputs.

The values for $\delta$ and $\psi$ determined from the decomposition of the measured Mueller matrices in both nonscattering (no microspheres) and turbid ( $\mu_{s}=30 \mathrm{~cm}^{-1}, g=0.95$ ) optically active $\left(\chi=1.96 \mathrm{deg} \mathrm{cm}^{-1}\right)$ phantoms with increasing birefringence (sample extension of 0 to $4 \mathrm{~mm}, \delta=0$ to $1.34 \mathrm{rad}$ ), are displayed in Fig. 8(a). ${ }^{37}$ The values from both clear and scattering samples are observed to be in close agreement with the controlled experimental inputs $(\psi \approx 1.96 \mathrm{deg}$ and $\delta \approx 1.34 \mathrm{rad}$ at $4 \mathrm{~mm}$ of extension). This suggests that the depolarizing effects of multiple scattering have been properly isolated and accounted for. Figure 8(b) shows the derived linear retardance $\delta$ and optical rotation $\psi$ parameters, using Monte Carlo generated Mueller matrices and with chiral molecule concentration as the independent variable. ${ }^{38}$ Again, both the nonscattering and turbid values compare well to the input parameters $(\delta \approx 1.4 \mathrm{rad}$ and $\psi \approx 1.96 \mathrm{deg}$ at $1 \mathrm{M}$ sucrose), showing self-consistency in inverse decomposition analysis and successful decoupling. The observed small increases in optical rotation in the presence of turbidity (in either the experimental phantoms or the analogous MC simulated turbid media) likely arise from the increase in optical pathlength due to multiple scattering (resulting in accumulations of $\psi$ values). ${ }^{37}$ Note that none of these trends could be gleaned from the original lumped Mueller matrix $\mathbf{M}$, where at best one would have to resort to semi-empirical comparison of changes in selected matrix elements, which contain complicating interrelated cross-talk contributions from several simultaneous effects.

The illustrative results presented above are for Mueller matrices recorded in the forward detection geometry. For conceptual and practical reasons, the extension of this approach to backward detection geometry (which is convenient for in situ measurements) is warranted, and has also been validated. ${ }^{99}$ Scatteringinduced artifacts are more coupled with the intrinsic medium polarization parameters in the backward detection geometry. At detection positions sufficiently close to the exact backscattering direction, contribution of the backscattered (singly or weakly scattered) photons can manifest themselves as large apparent scattering-induced linear retardance and diattenuation effects even from isotropic $(\Delta n=0)$ scattering medium; this masks the smaller effects due to, and thus accurate determination of, 


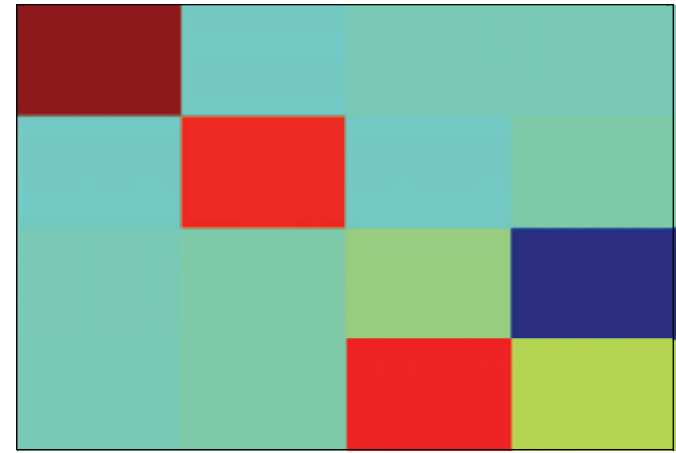

$\mathbf{M}_{\mathbf{R}}$

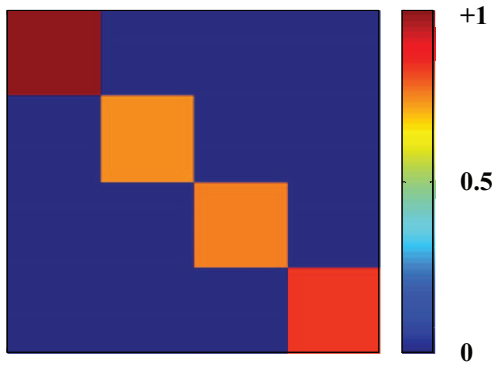

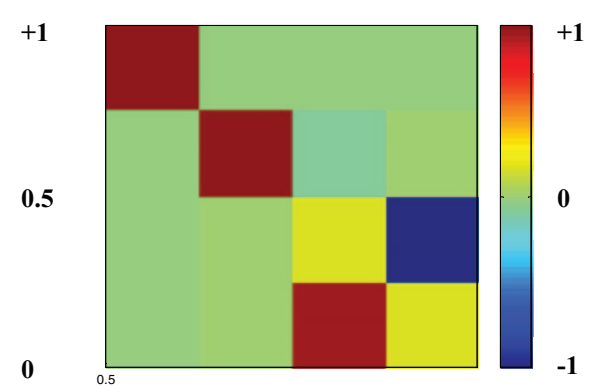

$\mathbf{M}_{\Delta}$

.
$+1$

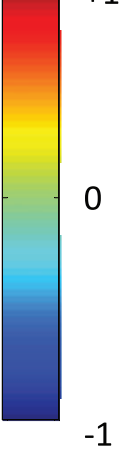

$-1$
$\mathbf{M}_{\mathbf{D}}$

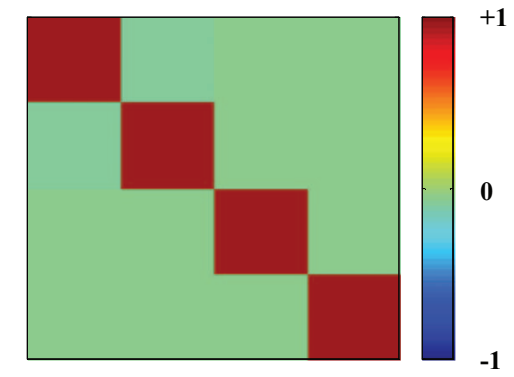

Fig. 7 Experimentally recorded Mueller matrix and the decomposed matrices for a birefringent (extension $=4 \mathrm{~mm}$ ), chiral (sucrose concentration $\left.=1 \mathrm{M}, \chi=1.96 \mathrm{deg} \mathrm{cm}^{-1}\right)$, turbid $\left(\mu_{s}=30 \mathrm{~cm}^{-1}, g=0.95\right.$, thickness $=1 \mathrm{~cm}$ ) phantom [the same phantom whose recorded Mueller matrix was presented in Fig. 5(a)]. The values for the individual elements of the decomposed basis matrices and the extracted polarization parameters are listed in Table 1.

intrinsic tissue parameters of interest such as $d, \delta, \theta$, and $\psi$. However, our studies have shown that as one moves away from the exact backscattering direction, the magnitude of scatteringinduced diattenuation and retardance gradually diminish and become weak (magnitudes $d \leq 0.05, \delta \leq 0.1$ ) for detection positions located at distances larger than a transport length away from the point of illumination $\left[r>l_{\text {tr }}, l_{\text {tr }}\right.$ is the transport scattering length $\left.=1 / \mu_{s}(1-g)\right]$. Thus, one method for simultaneous determination of the intrinsic values of all the polarization parameters from a turbid medium in the backward detection geometry is to perform measurements at a distance larger than a transport length away from the point of illumination. ${ }^{99}$

Our continuing validation studies on tissue-like complex turbid media (whose constituent properties are known and usercontrolled a priori) with both experimental and MC-simulated Mueller matrices have demonstrated that turbid media having weak diattenuation (magnitude $d \leq 0.1$ ), the decompositionderived polarization parameters are approximately independent of the selected multiplication order of the basis matrices in the decomposition process [see discussion following Eq. (50)], and also represent true medium properties. ${ }^{96}$ This is important in tissue: although many biological molecules (such as amino acids, proteins, nucleic acids) exhibit dichroism or diattenuation, the overall magnitude is much lower compared to the other polarimetry effects. The extracted polarization parame- ters are therefore expected to be independent of the selected multiplication order of the basis matrices in actual tissues. Table 2 gives an example of such decomposition analysis performed on Mueller matrix recorded from dermal tissue of an athymic nude mouse. ${ }^{100}$ The constituent basis matrices obtained via decomposition orders of Eq. (50a) $\left(\mathbf{M} \Leftarrow \mathbf{M}_{\Delta} \mathbf{M}_{\mathbf{R}} \mathbf{M}_{\mathbf{D}}\right)$ and Eq. (50d) $\left(\mathbf{M} \Leftarrow \mathbf{M}_{\mathbf{D}} \mathbf{M}_{\mathbf{R}} \mathbf{M}_{\Delta}\right)$ and the corresponding values of the extracted polarization parameters are shown in the table. The similarity in the elements of the basis matrices derived through the two-selected multiplication orders (other four possible orders being ruled out in light of physical or mathematical considerations ${ }^{95,96}$ ), suggests that the polarimetry events in actual tissue does not occur in a preferred sequence, but rather simultaneously as expected. Importantly, the excellent agreement between the polarization parameters extracted via the two decomposition orders and the true parameter values underscores the validity of the decomposition formalism and its self-consistency with respect to the potential ambiguity of ordering.

Decoupling and quantification of the individual intrinsic tissue polarimetry characteristics is thus enabled by the polar decomposition analysis despite their simultaneous occurrence, even in the presence of numerous complexities due to multiple scattering. The ability to isolate individual polarization properties provides a potentially valuable noninvasive tool for 
Table 1 The values for the polarization parameters extracted from the decomposed matrices (2nd column), for the experimental Mueller matrix shown in Figs. 7 and 5(a). The input control values for linear retardance $\delta$ and optical rotation $\psi$ (3rd column) were obtained from measurement on a clear $\left(\mu_{\mathrm{s}}=0 \mathrm{~cm}^{-1}\right)$ phantom having the same extension $(=4 \mathrm{~mm})$ and similar concentration of sucrose $(1 \mathrm{M})$ as that of the turbid phantom, and corrected for the increased pathlength due to multiple scattering (determined from Monte Carlo modeling). The expected value for the net depolarization coefficient $\Delta$ was determined from the Monte Carlo simulation of the experiment. (Adopted from Ref. 37).

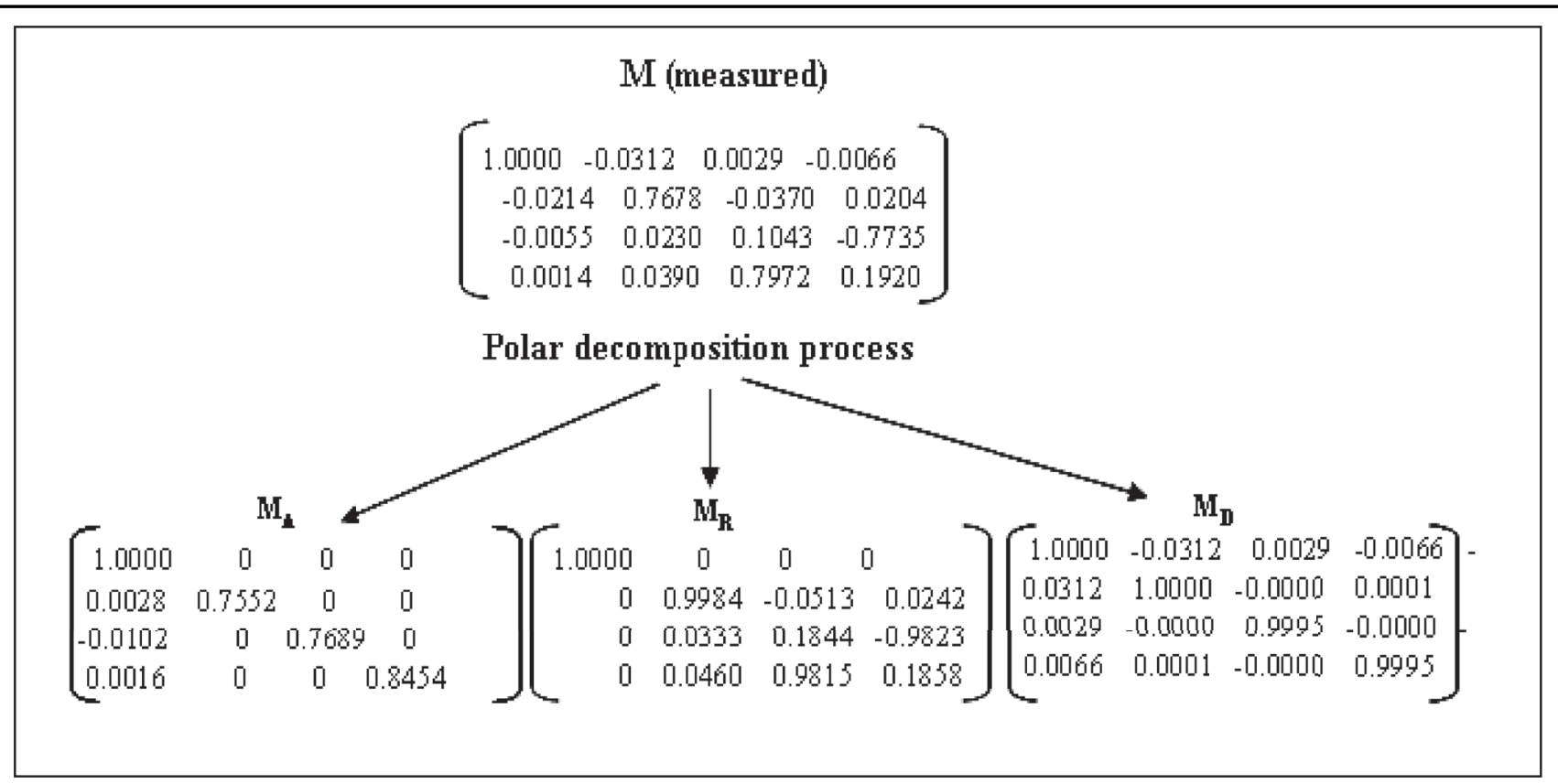

biological tissue examinations. In the following, we discuss ongoing and prospective biomedical applications of the intrinsic tissue polarimetry characteristics.

\subsection{Biomedical Applications of the Intrinsic Tissue Polarimetry Characteristics}

\subsubsection{Quantitative Stokes-Mueller polarimetry for glucose sensing}

Noninvasive glucose monitoring in diabetic patients remains one of the most important unsolved problems in modern medicine. Unfortunately, the most reliable current method for glucose monitoring necessitates the drawing of blood, usually by a finger prick several times a day-a painful, inconvenient, and poorly compliant procedure. A tremendous need therefore exists for a noninvasive glucose monitoring method, with relevant biopho- tonics research in NIR spectroscopy, Raman spectroscopy, fluorescence, optical coherence tomography, photoacoustics, and polarimetry. ${ }^{24} \mathrm{~A}$ common difficulty with the various proposed noninvasive techniques is the indirect, and often weak, relationship between the change in the measured signal and the corresponding change in the absolute glucose levels. Polarimetry, based on the chiral (handed) nature of the glucose molecules and its associated optical activity, is promising as it is potentially specific to glucose. Although such measurements in clear media have been used for decades in the sugar and food industries, polarimetric attempts for glucose quantification in a complex random medium like tissue have to overcome the following challenges: i. light is highly depolarized upon tissue multiple scattering, so detection of a polarization-preserved signal from which to extract the small glucose-induced optical rotation (physiological glucose blood concentration is 5 to $10 \mathrm{~mm}$ (somewhat greater range in diabetics, resulting in optical rotation in the 
(a)

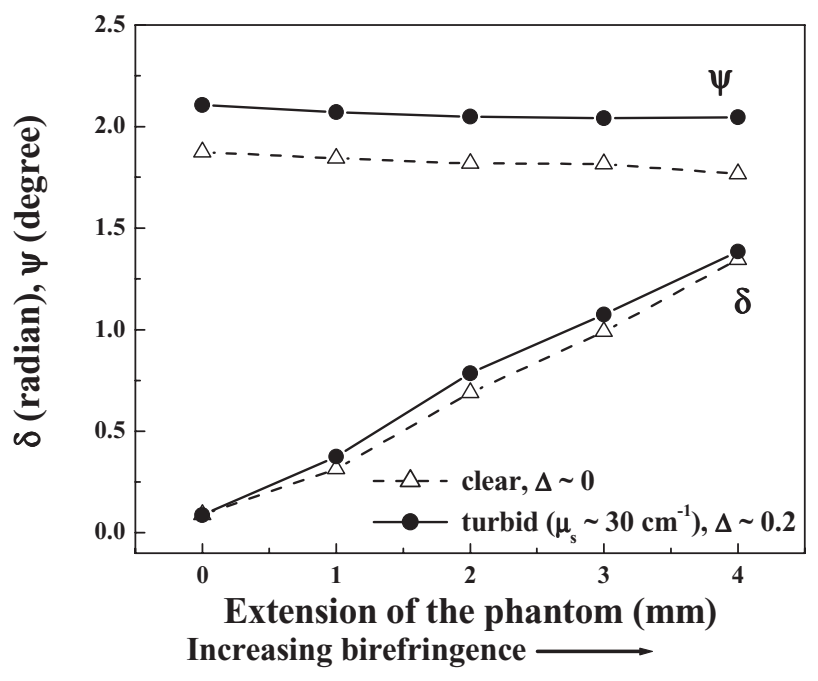

(b)

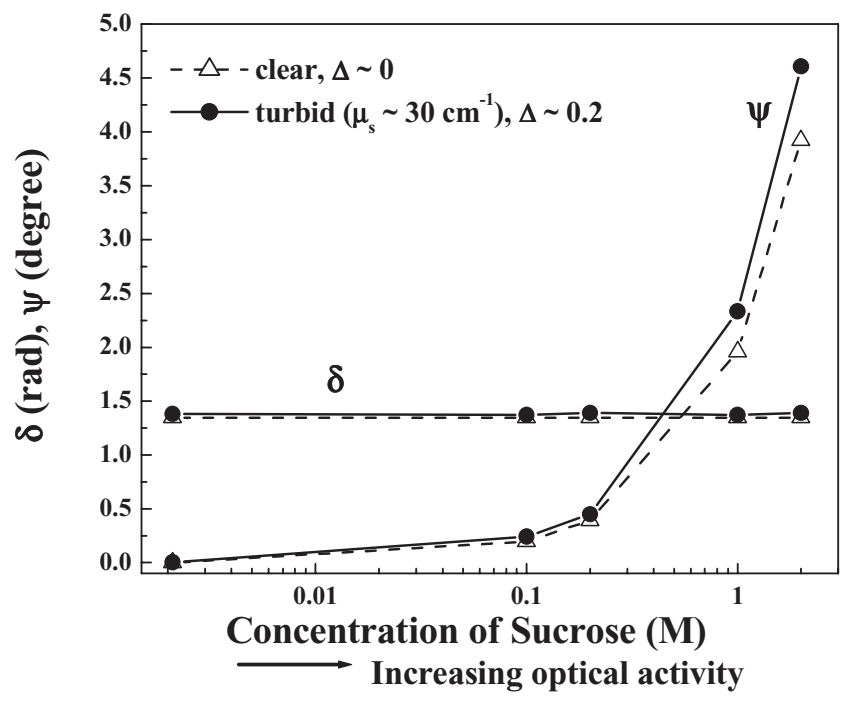

Fig. 8 (a) Linear retardance $\delta$ and optical rotation $\psi$ estimated from the decomposition of experimentally recorded Mueller matrices from solid chiral $\left(\chi=1.96 \mathrm{deg} \mathrm{cm}^{-1}\right.$, corresponding to $1 \mathrm{M}$ concentration of sucrose) phantoms having varying degrees of strain-induced birefringence (extension of 0 to $4 \mathrm{~mm}, \delta=0$ to $1.345 \mathrm{rad}$ ). Results are shown for both clear $\left(\mu_{s}=0 \mathrm{~cm}^{-1}\right)$ and turbid $\left(\mu_{s}=30 \mathrm{~cm}^{-1}, \mathrm{~g}\right.$ $=0.95$ ) phantoms. The values for the decomposition-derived depolarization coefficient $\Delta$ are also noted. The measurements were performed in the forward direction $(\gamma=0 \mathrm{deg})$ through a $1 \times 1 \times 4 \mathrm{~cm}^{3}$ phantom. The symbols are experimental data and the lines are guide for the eye. (Adopted from Refs. 15 and 38.) (b) The values for $\delta$ and $\psi$ estimated from the decomposition of Monte Carlo-generated Mueller matrices from birefringent media (linear birefringence $\Delta n=1.36 \times 10^{-5}$, corresponding to $\delta=1.345 \mathrm{rad}$ for a pathlength of $1 \mathrm{~cm}$, the axis of linear birefringence was kept along the vertical direction, orientation angle $=90 \mathrm{deg})$ having varying levels of chirality $(\chi=0,0.196,0.392,1.96$, and $3.92 \mathrm{deg} \mathrm{cm}^{-1}$, corresponding to concentration of sucrose of 0 , $0.1,0.2,1$, and $2 \mathrm{M}$, respectively). Results are shown for both clear ( $\mu_{s}$ $\left.=0 \mathrm{~cm}^{-1}\right)$ and turbid $\left(\mu_{s}=30 \mathrm{~cm}^{-1}, \mathrm{~g}=0.95\right)$ media. The symbols are data and the lines are guides for the eyes. (Adopted from Refs. 15 and 38.) milli-degree levels) is a formidable challenge; ii. simultaneous occurrence of several polarization effects hinders the isolation and quantification of the small glucose related signal (for example, the often much larger changes in the orientation angle of the polarization vector due to scattering can easily mask the small glucose-induced rotation); ${ }^{14,31,64}$ iii. other optically active chiral species are present in tissue, thus contributing to the observed optical rotation and hiding/confounding the specific glucose contribution.

With regard to addressing the first problem, we and others have shown that even in the presence of severe depolarization, measurable polarization signals can be reliably obtained from scattering media such as biological tissue. ${ }^{31-36}$ In fact, the high-sensitivity polarization modulation/synchronous detection experimental system, described in Sec. 4, was specifically developed for measuring small polarization signals in the presence of large depolarized background of multiply scattered light. Figure 9(a) gives an example on the potential for measuring very small optical rotations (milli-degree levels) in turbid media using the sensitive polarization modulation/synchronous detectionbased polarimetry system (described in Sec. 3.1). ${ }^{32}$ Measured glucose-induced optical rotation in scattering phantoms $(1.4-\mu \mathrm{m}$ diameter polystyrene microspheres in water, resulting scattering coefficient of $\mu_{s} \sim 28 \mathrm{~cm}^{-1}$ ) as calculated from Mie theory (exact value dependent on glucose concentration due to the latter's refractive index matching effect) with added glucose concentrations down to physiological levels (5 to $10 \mathrm{~mm}$ ) are shown in this figure. These measurements were performed in the forward direction [detection angle $\gamma=0$ deg in Fig. 9(a)] through $1 \mathrm{~cm}$ of scattering material $\left(1 \times 1 \times 4 \mathrm{~cm}^{3}\right.$ quartz cuvette containing the turbid chiral suspensions). A moderate scattering level was selected $\left(\sim 1 / 3\right.$ of biological tissue in the visible-near IR range $\left.{ }^{50}\right)$, as depolarization in the forward direction through thick samples ( $1 \mathrm{~cm}$ in this case) is quite severe, limiting the accuracy with which small optical rotation values due to small glucose levels can be accurately measured. The optical rotation values were calculated from the measured Stokes parameters as

$$
\psi=\frac{1}{2} \tan ^{-1}\left(\frac{u}{q}\right)
$$

Note that the scattering/detection geometry-induced artifacts are minimal in the forward direction, but can be significantly greater at other detection directions (highest in the exact backscattering direction $\gamma=180 \mathrm{deg}) .{ }^{64}$ Yet the backward detection geometry is more convenient for in situ measurements. It is thus essential to isolate the optical rotation caused exclusively by chiral molecules from the (often much larger) apparent rotation caused by the scattering/detection geometry effects. The Mueller matrix decomposition methodology, described in the preceding section, is indeed able to perform this task, as shown in Fig. 9(b).$^{38}$ The variation of scattering induced rotation in the backscattering direction is displayed as a function of distance from the point of illumination of a chiral $\left(\chi=0.082 \mathrm{deg} \mathrm{cm}^{-1}\right.$, corresponding to $100 \mathrm{~mm}$ concentration of glucose), nonbirefringent $(\Delta n=0)$, turbid medium $\left(\mu_{s}=30 \mathrm{~cm}^{-1}\right.$, thickness $=1 \mathrm{~cm})$. The incident light is horizontally $(0 \mathrm{deg})$ polarized (Stokes vector $\left[\begin{array}{llll}1 & 1 & 0 & 0\end{array}\right]^{\mathrm{T}}$ ), and the rotation was calculated from the recorded Stokes parameters of light exiting the sample through the backscattering plane $(X-Y$ plane, $Z=0)$, using Eq. (51). As seen, changes in the polarization caused by scattering 
Table 2 Top: The experimental Mueller matrix and the decomposed basis matrices from dermal tissue of an athymic nude mouse. The Mueller matrix was recorded in-vivo from a dorsal skinfold window chamber mouse model, using a high sensitivity turbid-polarimetry system. The constituent basis matrices obtained via decomposition with the order of Eq. (50a) $\left(\mathbf{M} \Leftarrow \mathbf{M}_{\Delta} \mathbf{M}_{\mathbf{R}} \mathbf{M}_{\mathbf{D}}\right)$ and Eq. (50d) $\left(\mathbf{M} \Leftarrow \mathbf{M}_{\mathbf{D}} \mathbf{M}_{\mathbf{R}} \mathbf{M}_{\Delta}\right)$ are shown in the 2 nd and the 3 rd rows respectively. Bottom: The values for the polarization parameters extracted from the decomposed matrices. Note that this is the first demonstration of Mueller matrix decomposition in live tissues (Ref. 100).

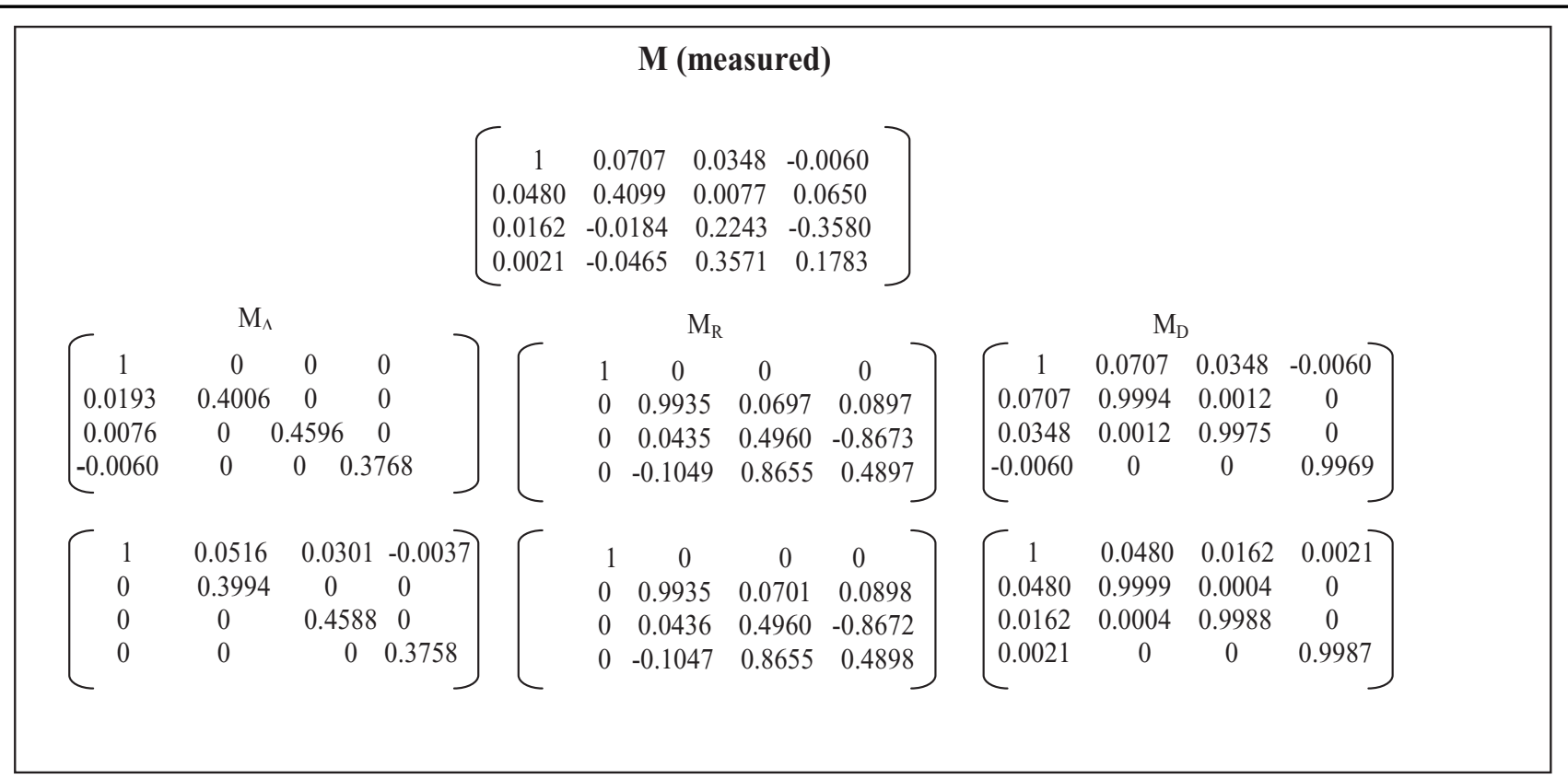

\begin{tabular}{|c|c|c|}
\hline Parameters & $\begin{array}{c}\text { Derived parameters using } \\
\text { the order of Eq. (50a) }\end{array}$ & $\begin{array}{c}\text { Derived parameters using } \\
\text { the order of Eq. (50d) }\end{array}$ \\
\hline $\mathrm{d}$ & $\mathbf{0 . 0 7 9}$ & $\mathbf{0 . 0 5 1}$ \\
\hline$\delta$ & $1.06 \mathrm{rad}$ & $1.058 \mathrm{rad}$ \\
\hline$\psi$ & $\mathbf{0 . 5 1} \mathbf{~ d e g}$ & $\mathbf{0 . 5 0} \mathbf{~ d e g}$ \\
\hline$\Delta$ & 0.58 & 0.59 \\
\hline
\end{tabular}

can manifest themselves as large apparent optical rotation. Decomposition analysis reveals that the large apparent rotation is due to the combined effect of scattering and linear diattenuation (also shown in the figure), yet this chirality-unrelated large signal can be successfully decoupled from the much smaller $\psi$ rotations of interest due by glucose. The Mueller matrix decomposition method thus has the potential to solve a major stumbling block for the polarimetric attempts for noninvasive glucose measurements in tissue. However, in order to realize this, one also has to address the issue of the confounding effects of the other optically-active molecules present in tissue. One method to tackle this problem is the spectral polarization measurements (that measures the optical rotatory dispersion of glucose). ${ }^{24} \mathrm{In}$ combination with MC-determined pathlength distributions, we and others are currently investigating spectroscopic-based po- larimetry combined with chemometric regression analysis to isolate the rotation due to glucose from that caused by other chiral tissue constituents. ${ }^{101}$

As for any new noninvasive glucometric methodology, noninvasive measurements of glucose levels (with the requisite sensitivity/specificity/accuracy) in actual tissues using the polarimetric approaches will have to be rigorously investigated. Certainly, the low physiological glucose levels, the high (and variable) levels of tissue scattering, the varying levels of tissue optical absorption, the presence of other optically-active molecules, and the confounding effects of various biological variables $(\mathrm{pH}$, temperature, etc.) will pose significant challenges to any noninvasive glucose monitoring approach, including the polarimetric approaches. Nevertheless, turbid polarimetry has shown early promise for future in vivo glucometry developments. It is also 
(a)

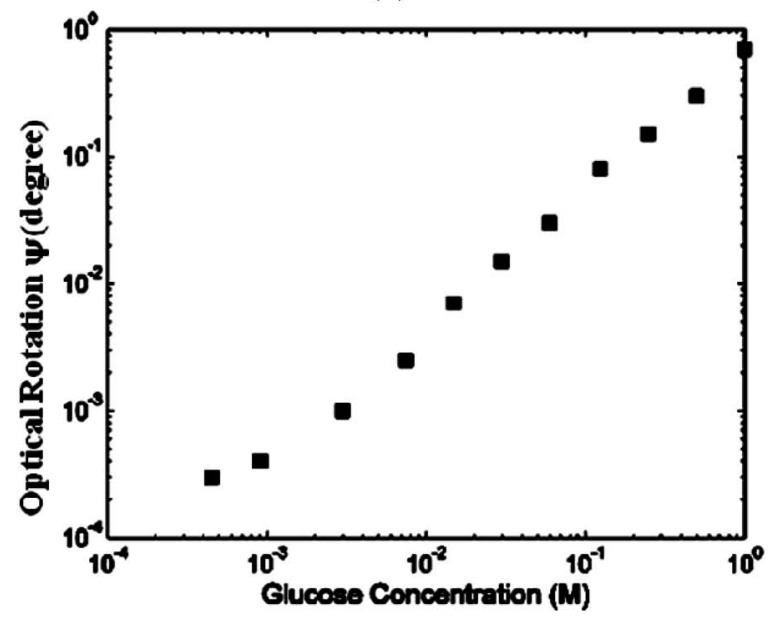

(b)

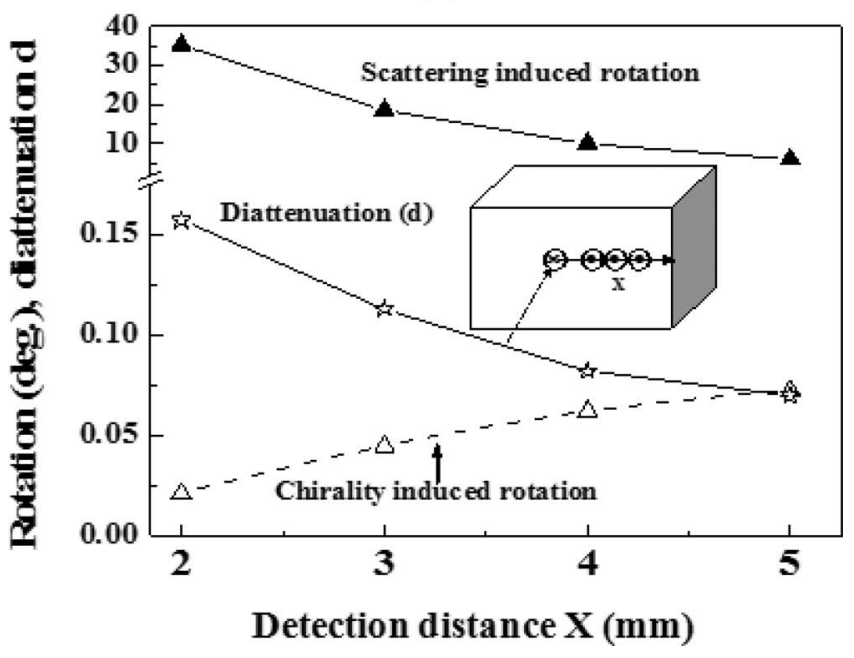

Fig. 9 (a) Logarithmic plot of optical rotation as a function of glucose concentration in scattering media $(1.4-\mu \mathrm{m}$ diameter polystyrene microspheres in water, $\mu_{S} \sim 28 \mathrm{~cm}^{-1}$ ) down to physiological glucose levels. Measurements were performed in the forward direction $(\gamma$ $=0 \mathrm{deg}$ ) through $1 \mathrm{~cm}$ of turbid media in a quartz cuvette. (Adopted from Refs. 16 and 32.) (b) Calculated optical rotation $\psi$ (derived from the decomposition of Monte Carlo generated Mueller matrices, solid circles) of scattered light emerging in the backscattering direction as a function of distance from the center of the incident beam of a chiral $(x=0.082 \mathrm{deg} / \mathrm{cm}$, corresponding to $100 \mathrm{~mm}$ concentration of glucose) isotropic turbid medium $\left(\mu_{s}=30 \mathrm{~cm}^{-1}, g=0.95\right.$, thickness $t=1 \mathrm{~cm}$ ). The corresponding scattering-induced rotation of the polarization vector derived from the Stokes parameters of scattered light (for incident Stokes vector $\left[\begin{array}{llll}1 & 0 & 1 & 0\end{array}\right]^{\top}$ ) is shown by open circles. The inset shows the backwards detection geometry. The chirality-induced rotation approaches zero in the exact backscattering direction $(X=0$, data not shown). The symbols are data and the lines are guides for the eyes. (Adopted from Refs. 15 and 38.)

likely that the solution for noninvasive glucose monitoring lies in multimodality approaches, for example, by combining polarimetry with another method (e.g., NIR spectroscopy), judiciously selecting their complementary strengths to overcome the formidable biological complexity inherent in this important clinical problem.

\subsubsection{Quantification of tissue structural anisotropy: representative applications in tissue diagnosis and regenerative therapy monitoring}

As noted previously, the presence of organized fibrous structures (collagen, elastin, myosin) in tissue manifests as structural anisotropy that can be probed using polarization birefringence measurements. Since the structural and functional properties of these fibers change as a result of tissue abnormalities or in response to treatment, birefringence measurement may offer a sensitive probe for assessing tissue status. A number of investigations have therefore addressed such polarization birefringence measurement for the detection of tissue abnormalities like osteoarthritis, thermal injury and cancer (e.g., basal and squamous cell carcinomas). ${ }^{102-105}$ Both Stokes vector and Mueller matrix analysis have been explored in these studies. Clearly, the decomposition methodology described previously, capable of decoupling small birefringence alterations from the other confounding polarization effects present in the composite signals of the measured Mueller matrix elements, is advantageous in this regard. In the following, we present an illustrate example of this novel polarimetry approach for monitoring regenerative treatments of the heart.

Healthy myocardial (heart) tissue is highly fibrous and anisotropic; accordingly it exhibits measurably high levels of linear birefringence. Upon suffering an infarction, a portion of the myocardium is deprived of oxygenated blood; subsequently cardiomyocytes die and are replaced by the fibrotic collagen (scar) tissue, often arranged in a random/chaotic fashion. Stem cell regenerative treatments have been shown to alter this remodeling process, by increasing the muscular and decreasing the scar tissue components. ${ }^{106}$ Accordingly, changes in the structure of the myocardium associated with infarction and treatment-induced remodeling alter the anisotropy of the tissue, and its measurement may offer a sensitive probe into the state of the myocardium after infarction and report on the success of regenerative treatments. Figure 10 summarizes the results of transmission Mueller matrix measurement and its decomposition, performed on 1-mm thick ex vivo myocardial samples from Lewis rats after myocardial infarction, both with and without stem cell treatments. ${ }^{38}$ Measurements were made using both the point measurement and imaging polarimetry systems. The point measurement system employed polarization modulation and synchronous lock-in detection as outlined in Sec. 3. The imaging polarimetry employed dc measurements with the PSGPSA-based approach (also discussed in Sec. 3) to construct the Mueller matrix. ${ }^{107}$

The Mueller matrices measured by either of these systems were analyzed via polar decomposition to obtain linear retardance $(\delta)$ values, as also summarized in Fig. 10. A large decrease in the magnitude of $\delta$ is seen in the infarcted region of the untreated myocardium [Fig. 10(b)]. In contrast, in the infarcted region after stem cell treatment an increase in $\delta$ toward the native levels can be observed, indicating regrowth and reorganization of the myocardium. The polarimetry images [Fig. 10 (c)] from the same tissue also show similar retardance trends as seen in the point measurement results, although some variation is seen (due to difference in measurement geometry and spatial heterogeneity in tissue optics). In the spatial variation of the retardance images, not only do the values change from 
(a)
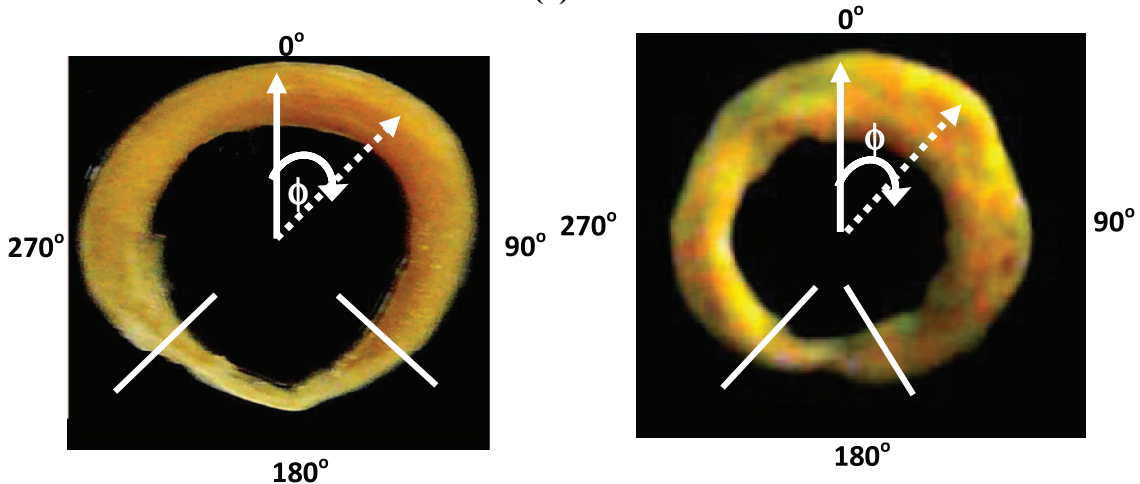

(b)

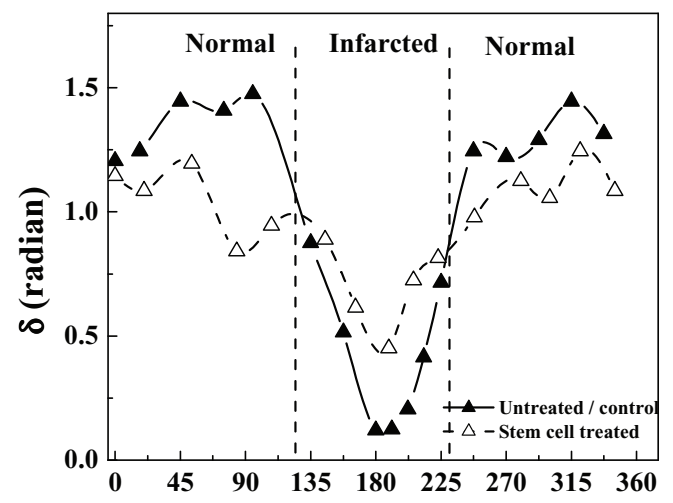

(c)
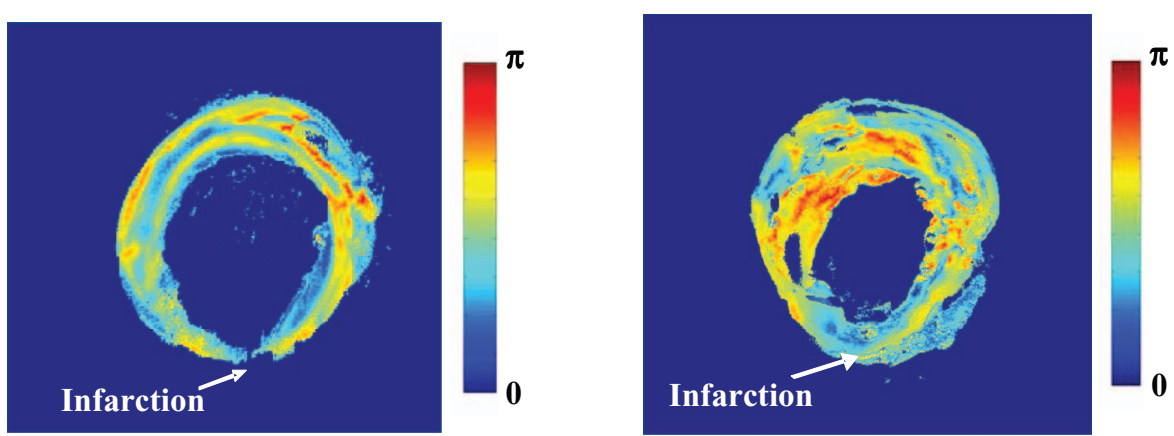

Fig. 10 Linear retardance $\delta$ derived from Mueller matrix measurements in 1-mm-thick tissue sections from Lewis rat hearts following myocardial infarction. (a) White-light photographs of untreated (left panel) and stem cell treated (right panel) tissue. The marked sectors around $\varphi=180$ deg show the infarcted regions. (b) Mueller matrix derived linear retardance $(\delta)$ values at different angular positions from the untreated (solid triangles) and treated (open triangles) samples. (c) The corresponding linear retardance $(\delta)$ images for the same tissue derived from the imaging polarimetry measurements. (Adopted from Refs. 15 and 38.)

infarct to normal, but within each region as well. In general, the $\delta$ values are higher in the middle of the myocardial wall with gradual lowering toward the edges. This variation through the myocardial wall is likely due to the change in orientation of the myocyte fibers, as the fiber orientation has been shown to undergo a rotation of $180 \mathrm{deg}$ from the outside of the ventricle to the inside. This variation through the myocardial wall is likely due to the change in orientation of the myocyte fibers through the wall. Note that the fiber orientation undergoes a rotation of $180 \mathrm{deg}$ from the outside of the ventricle to the inside. While on the inside and outside of the ventricle the fibers are oriented per- pendicular to the axial imaging plane they lie in it in the central region. Since the axis of birefringence (direction of the fibers) is along the direction of the light's propagation, the corresponding retardance values in the outside and inside wall are generally lower. The opposite is true in the central region (birefringence axis is perpendicular to light propagation direction), leading to larger magnitude of the observed $\delta .{ }^{38}$

Methods for dealing with these orientation complications are described below; nevertheless, further analysis of the date in Fig. 10 revealed statistically-significant differences in derived retardance values between normal and infarcted regions, and 
between infarcted regions with and without stem-cell treatments. ${ }^{38}$ The retardance was significantly reduced in the region of infarction and increased through the marginal region to higher levels observed in unaffected tissues. The increase in $\delta$ in the infarcted regions of the stem-cell treated hearts indicated reorganization and regrowth of the myocardium (as also confirmed by histologic examination). These results demonstrated the ability of tissue polarimetry to characterize the micro-organizational state of the myocardium via its measured anisotropy, and the potential of this approach for monitoring regenerative treatments of myocardial infarction. A study with nonlinear microscopy (second harmonic generation, two-photon excited fluorescence) has recently been performed to validate the polarimetry results, and to probe the underlying causes of the measured retardance signals, in the context of collagen versus cardiomyocytes components and their spatial organization. ${ }^{107}$

Note that the derived linear retardance values $\delta$ are related to the underlying tissue birefringence $\left(\Delta n=n_{e}-n_{o}\right)$, via $\delta=(2 \pi / \lambda) \Delta n L$, where $L$ is the pathlength. However, the apparent retardance (measured) is not only a function of the intrinsic anisotropy of tissue, but also depends on the experimental geometry. This follows because as photons propagate in the medium, the difference in refractive indices $\left[n(\vartheta)-n_{o}\right]$ experienced by them depends on their propagation direction with respect to the extraordinary axis, as described in Eq. (38). Anisotropic tissues may well exhibit complex and changing orientations of their birefringent spatial domains, so the measured linear retardance is expected to be significantly influenced by the orientation of the sample with respect to the probing beam [as is apparent from the results of polarimetry imaging presented in Fig. 10(c)]. Attempts have therefore been made to develop methods to extract geometry-independent metrics of tissue anisotropy (intrinsic birefringence). We have recently explored a dual projection polarimetry method to tackle this problem, ${ }^{108}$ whereby the sample is imaged twice at different incident angles of the probing beam. The apparent linear retardance $\delta_{\text {app }}$ and azimuthal angle $\vartheta$ (the projection of the anisotropy axis in the plane of imaging), measured with two different sample/beam geometries, provide sufficient information to reconstruct the true intrinsic magnitude and orientation angle of the retardance. After successful validation of this approach on birefringent spherical phantoms, the method was initially explored for the measurement (imaging) of the anisotropy axis and its true magnitude in ex vivo porcine myocardium tissues. ${ }^{109}$ Polarimetry-derived magnitude and orientation of tissue anisotropy should prove useful in assessing the effects of disease progression and treatment monitoring scenarios. Note, however, that the reconstruction process adopted in this method assumes constant uniaxial orientation of tissue birefringence. Polarization transfer in tissues with orientationvarying uniaxial spatial domains, or potentially in nonuniaxial (biaxial) tissues is a formidable challenge that is being actively investigated.

In addition to linear birefringence and optical activity, the depolarization properties of tissue also contain useful morphological information on the biological scattering structures (such as their density, size, distribution, shape, and refractive index). Thus, even the depolarization properties, if properly quantified and analyzed, can be exploited for quantitative tissue diagnosis and characterization. Indeed, interesting differences have been reported in the Mueller matrix-derived depolarization coeffi- cient $(\Delta)$ between the cancerous and the normal sites from oral cavity ${ }^{104,110}$ and cervical tissues. ${ }^{105,111}$ Studies are also underway to link the measured depolarization properties to the optical transport parameters $\left(\mu_{a}, \mu_{s}\right.$ and $g$ ), as a direct link to the underlying scattering properties of tissue.

The Mueller matrix decomposition method has also been explored for quantification of structural changes in skeletal muscle, which is one of the most abundant tissues in human body and exhibits strong birefringence effects due to well organized fibrous structures. ${ }^{112}$ The decomposition-derived polarization properties (and their respective images) indeed revealed characteristic patterns of muscle organization. ${ }^{112}$ The effect of the microstructural architecture of skeletal muscles on the recorded Mueller matrix elemental images have also been theoretically studied by incorporating a sphere-cylinder scattering model (SCSM) in polarization sensitive Monte Carlo simulations. ${ }^{113}$ The results demonstrated that the SCSM model can successfully reproduce most of the important characteristic features of experimental Mueller matrices of the skeletal muscle tissues, and thus this can be used to study the interactions of polarized light with complex tissue structures such as skeletal muscles.

To summarize, this section has illustrated the biomedical utility of the various intrinsic tissue polarimetry characteristics with representative examples. A novel general polarimetry method for inverse signal analysis that can be applied to complex tissue polarimetry data to isolate specific quantities of interest has been described with selected validation results. Initial finding in two scenarios of significant clinical interest, noninvasive glucose measurements and monitoring of regenerative treatments of the heart, have been presented. Clearly there are many other ongoing and prospective applications, both in tissue diagnostics and in treatment response monitoring. Although significant challenges remain, the tissue polarimetry progress to date bodes well for future in vivo biomedical deployments. It should also be mentioned here that polarization sensitive optical coherence tomography (PS-OCT) (that combines the depth sectioning capability of conventional optical coherence tomography with polarization resolved measurements) is another polarimetric method that has received particular attention and has already yielded very promising results in various diagnostic applications. This has not been discussed in this review. For details of the principle and the various experimental approaches used in PS-OCT and their applications, the reader is referred to Ref. 114.

\section{Concluding Remarks}

In this tutorial review, the use of polarized light for biological tissue assessment has been discussed, in the context of tissue imaging and in the context of characterization/diagnosis. As seen, the developed experimental and theoretical methodologies are improving and moving toward quantification, as is essential for meaningful advances in this challenging field. Early studies in polarization-gated tissue imaging, birefringence monitoring, and chirality detection illustrate the advantages of polarimetry over the corresponding polarization-blind intensity-based methods, but also point out significant polarimetric challenges. Our understanding of the complex polarization effects in tissues and methods of their quantification are continually improving. Future research to improve polarization tissue imaging and 
diagnostic characterization will hopefully be informed by these developments. There is clearly much work still to be done in the field of tissue polarimetry, but the prospects of meaningful in vivo deployment appear promising.

\section{References}

1. V. Ronchi, The Nature of Light, Harvard University Press, Cambridge, Massachusetts (1970).

2. J. Michl and E. W. Thulstrup, Spectroscopy with Polarized Light, VCH, New York (1986).

3. R. A. Hegstrom and D. K. Kondepudi, "The handedness of the universe," Sci. Am. 262, 108-115 (1990).

4. C. A. Browne and F. W. Zerban, Physical and Chemical Methods of Sugar Analysis, Wiley, New York (1941).

5. B. Jirgensons, Optical Rotatory Dispersion of Proteins and Other Macromolecules, New York, USA (1969).

6. D. S. Kliger, J. W. Lewis, and C. E. Randall, Polarized Light in Optics and Spectroscopy, Academic Press-Harcourt Brace Jovanovich, New York (1990).

7. C. Brouseau, Fundamentals of Polarized Light a Statistical Approach, Wiley, New York (1998)

8. M. Gadsden, P. Rothwell, and M. J. Taylor, "Detection of circularly polarized-light from noctilucent clouds," Nature 278, 628-629 (1979).

9. A. Chrysostomou, P. W. Lucas, and J. H. Hough, "Circular polarimetry reveals helical magnetic fields in the young stellar object HH 135-136," Nature 450, 71-73 (2007).

10. R. S. Gurjar, V. Backman, L. T. Perelman, I. Georgakoudi, K. Badizadegan, I. Itzkan, R. R. Dasari, and M. S. Feld, "Imaging human epithelial properties with polarized light scattering spectroscopy," Nat. Med. 7, 245-1249 (2001).

11. V. V. Tuchin, L. Wang, and D. À. Zimnyakov, Optical Polarization in Biomedical Applications, New York, USA (2006).

12. L. V. Wang, G. L Coté, and S. L. Jacques, "Special section guest editorial: tissue polarimetry," J. Biomed. Opt. 7, 278 (2002).

13. K. C. Hadley and I. A. Vitkin, "Optical rotation and linear and circular depolarization rates in diffusely scattered light from chiral, racemic, and achiral turbid media," J. Biomed. Opt. 7, 291-299 (2002).

14. S. Manhas, M. K. Swami, P. Buddhiwant, N. Ghosh, P. K. Gupta, and K. Singh, "Mueller matrix approach for determination of optical rotation in chiral turbid media in backscattering geometry," Opt. Express. 14, 190-202 (2006).

15. N. Ghosh, M. Wood, and A. Vitkin, "Polarized light assessment of complex turbid media such as biological tissues using Mueller matrix decomposition," Chapter 9, Handbook of Photonics for Biomedical Science, V. V. Tuchin, Ed., pp. 253-282, Taylor and Francis Publishing, London (2010).

16. M. F. G. Wood, N. Ghosh, X. Guo and I. A. Vitkin, "Towards noninvasive glucose sensing using polarization analysis of multiply scattered light," Chap. 17, Handbook of Optical Sensing of Glucose in Biological Fluids and Tissues, V. V. Tuchin, Ed., Series in Medical Physics and Biomedical Engineering, Vol. 12, Taylor and Francis Publishing, London (2008).

17. S. L. Jacques, J. C. Ramella-Roman, and K. Lee, "Imaging skin pathology with polarized light," J. Biomed. Opt. 7, 329-340 (2002).

18. E. Collette, Polarized light: Fundamentals and Applications, Marcel Dekker, Inc., New York (1990).

19. C. F. Bohren and D. R. Huffman, Absorption and Scattering of Light by Small Particles, Wiley, New York (1983).

20. R. A. Chipman, "Polarimetry," Chap. 22 in Handbook of Optics, 2nd Ed., M. Bass, Ed., Vol. 2, pp. 22.1-22.37, McGraw-Hill, New York (1994).

21. W. S. Bickel and W. M. Bailey, "Stokes vectors, Mueller matrices, and polarization of scattered light," Am. J. Phys. 53, 468-478 (1985).

22. J. J. Gil, "Characteristic properties of Mueller matrices," J. Opt. Soc. Am. A 17, 328-334 (2000).

23. S. Yau Lu and R. A. Chipman, "Interpretation of Mueller matrices based on polar decomposition," J. Opt. Soc. Am. A 13, 1106-1113 (1996).

24. R. J. McNichols and G. L. Cote, "Optical glucose sensing in biological fluids: an overview," J. Biomed. Opt., 5, 5-16 (2000).
25. E. Collett, "Measurement of the four Stokes polarization parameters with a single circular polarizer," Opt. Commun. 52, 77-80 (1984).

26. V. Sankaran, K. Schonenberger, J. T. Walsh, Jr., and D. J. Maitland, "Polarization discrimination of coherently propagating light in turbid media," Appl. Opt. 38, 4252- 4261 (1999).

27. V. Sankaran, J. T. Walsh, Jr., and D. J. Maitland, "Comparative study of polarized light propagation in biological tissues," J. Biomed. Opt. 7, 300-306 (2002).

28. N. Ghosh, A. Pradhan, P. K. Gupta, S. Gupta, V. Jaiswal, and R. P. Singh, "Depolarization of light in a multiply scattering medium: effect of refractive index of scatterer," Phys. Rev. E 70, 066607 (2004).

29. N. Ghosh, P. K. Gupta, A. Pradhan, and S. K. Majumder, "Anomalous behavior of depolarization of light in a turbid medium," Phys. Lett. A 354, 236-242 (2006).

30. N. Ghosh, H. S. Patel, and P. K. Gupta, "Depolarization of light in tissue phantoms - effect of a distribution in the size of scatterers," Opt. Express 11, 2198-2205 (2003).

31. X. Guo, M. F. G. Wood, and I. A. Vitkin, "Angular measurement of light scattered by turbid chiral media using linear Stokes polarimetry," J. Biomed. Opt. 11, 041105 (2006).

32. D. Côté and I. A. Vitkin, "Balanced detection for low-noise precision polarimetric measurements of optically active, multiply scattering tissue phantoms," J. Biomed. Opt. 9, 213-220 (2004).

33. I. A. Vitkin, R. D. Laszlo, and C. L. Whyman, "Effects of molecular asymmetry of optically active molecules on the polarization properties of multiply scattered light," Opt. Express 10, 222-229 (2002).

34. R. C. N. Studinski and I. A. Vitkin, "Methodology for examining polarized light interactions with tissues and tissuelike media in the exact backscattering direction," J. Biomed. Opt. 5, 330-337 (2000).

35. J. Badoz, M. Billardon, J. C. Canit, and M. F. Russel, "Sensitive devise to measure the state and degree of polarization of a light beam using a birefringence modulator," J. Opt. 8, 373-384 (1977).

36. M. P. Silverman, N. Ritchie, G. M. Cushman, and B. Fisher, "Experimental configurations using optical phase modulation to measure chiral asymmetries in light specularly reflected from a naturally gyroptopic medium," J. Opt. Soc. Am. A 5, 1852-1863 (1988).

37. N. Ghosh, M. F. G. Wood, and I. A. Vitkin, "Mueller matrix decomposition for extraction of individual polarization parameters from complex turbid media exhibiting multiple scattering, optical activity and linear birefringence," J. Biomed. Opt. 13, 044036 (2008).

38. N. Ghosh, M. F. G. Wood, S.-H. Li, R. D. Weisel, B. C. Wilson, R.-K. $\mathrm{Li}$, and I. A. Vitkin, "Mueller matrix decomposition for polarized light assessment of biological tissues," J. Biophotonics 2, 145-156 (2009).

39. R. M. A. Azzam, "Photopolarimetric measurement of the Mueller matrix by Fourier analysis of a single detected signal," Opt. Lett. 2, 148-150 (1978).

40. D. H. Goldstein, "Mueller matrix dual-rotating retarder polarimeter," Appl. Opt. 31, 6676-6683 (1990).

41. M. H. Smith, "Optimization of a dual-rotating-retarder Mueller matrix polarimeter," Appl. Opt. 41, 2488-2493 (2002).

42. M. Dubreuil, S. Rivet, B. Le Jeune, and J. Cariou, "Snapshot Mueller matrix polarimeter by wavelength polarization coding," Opt. Express 15, 13660-13668 (2007).

43. J. S. Baba, J. R. Chung, A. H. DeLaughter, B. D. Cameron, and G. L. Cote, "Development and calibration of an automated Mueller matrix polarization imaging system," J. Biomed. Opt. 7, 341-348 (2002).

44. A. De Martino, E. Garcia-Caurel, B. Laude, and B. Drévillon, "General methods for optimized design and calibration of Mueller polarimeters," Thin Solid Films 455-456, 112-119 (2004).

45. B. Laude-Boulesteix, A. De Martino, B. Drévillon, and L. Schwartz, "Mueller polarimetric imaging system with liquid crystals," Appl. Opt. 43, 2824-2832 (2004).

46. M. Mujat and A. Dogariu, "Real-time measurement of the polarization transfer function," Appl. Opt. 40, 34-44 (2001).

47. E. Compain, S. Poirier, and B. Drevillon, "General and self-consistent method for the calibration of polarization modulators, polarimeters, and Mueller-matrix ellipsometers," Appl. Opt. 38, 3490-3502 (1999).

48. C.-Y. Han and Y.-F. Chao, "Photoelastic modulated imaging ellipsometry by stroboscopic illumination technique," Rev. Sci. Instrum. 77, 023107 (2006).

49. R. M. Azzam and N. M. Bashara, Ellipsometry and Polarized Light, North Holland, Amsterdam (1979). 
50. A. J. Welch, M. J. C. Van Germert, W. M. Star, and B. C. Wilson, "Overview of tissue optics," in Optical Thermal Response of Laser Irradiated Tissue, A. J. Welch and M. J. C. Van Germert, Eds., Plenum, New York (1995).

51. A. Ishimaru, Wave Propagation and Scattering in Random Media, Academic Press, New York (1978).

52. D. Bicout and C. Brosseau, "Multiply scattered waves through a spatially random medium: entropy production and depolarization," J. Phys. I (France) 2, 2047-2063 (1992).

53. D. Bicout, C. Brosseau, A. S. Martinez, and J. M. Schmitt, "Depolarization of multiply scattered waves by spherical diffusers: Influence of size parameter," Phys. Rev. E 49, 1767-1770 (1994).

54. E. E. Gorodnichev, A. I. Kuzolov, and D. B. Rozozkin, "Diffusion of circularly polarized light in a disordered medium with large scale inhomogeneities," JETP Lett. 68, 22-28 (1998).

55. A. D. Kim and M. Moscoso, "Influence of the refractive index on the depolarization of multiply scattered waves," Phys. Rev. E 64, 026612 (2001).

56. M. Moscoso, J. B. Keller, and G. Papanicolaou, "Depolarization and blurring of optical images by biological tissue," J. Opt. Soc. Am. A 18, 948-960 (2001).

57. L. F. Rojas-Ochoa, D. Lacoste, R. Lenke, P. Schurtenberger, and F. Scheffold, "Depolarization of backscattered linearly polarized light," J. Opt. Soc. Am. A 21, 1799-1804 (2004).

58. M. Xu and R. R. Alfano, "Random walk of polarized light in turbid media," Phys. Rev. Lett. 95, 213901 (2005).

59. D. A. Zymniakov, Y. P. Sinichkin, P. V. Zakharov and D. N. Agafonov, "Residual polarization of non-coherently backscattered linearly polarized light: the influence of the anisotropy parameter of the scattering medium," Waves Random Media 11, 395-412 (2001).

60. L. Wang, S. L. Jacques, and L. Zheng, "MCML-Monte Carlo modeling of light transport in multi-layered tissues," Comput. Methods Programs Biomed. 47, 131-146 (1995).

61. M. F. G. Wood, X. Guo, and I. A. Vitkin, "Polarized light propagation in multiply scattering media exhibiting both linear birefringence and optical activity: Monte Carlo model and experimental methodology," J. Biomed. Opt. 12, 014029 (2007).

62. M. Xu, "Electric field Monte Carlo for polarized light propagation in turbid media," Opt. Express 12, 6530-6539 (2004).

63. J. C. Ramella-Roman, S. A. Prahl, and S. L. Jacques, “Three Monte Carlo programs of polarized light transport into scattering media: part I," Opt. Express 13, 4420-4438 (2005).

64. D. Côté and I. A. Vitkin, "Robust concentration determination of optically active molecules in turbid media with validated threedimensional polarization sensitive Monte Carlo calculations," Opt. Express 13, 148-163 (2005).

65. R. C. Jones, "New calculus for the treatment of optical systems. VII. Properties of the N-matrices," J. Opt. Soc. Am. 38, 671-685 (1948).

66. J. C. Hebden, S. R. Arridge, and D. T. Delpy, "Optical imaging in medicine: 1. Experimental techniques," Phys. Med. Biol. 42, 825-840 (1997).

67. C. Dunsby and P. M. W. French, "Techniques for depth-resolved imaging through turbid media including coherence-gated imaging," J. Phys. D: Appl. Phys. 36, R207-R227 (2003).

68. J. M. Schmitt, A. H. Gandjbakhche, and R. F. Bonner, "Use of polarized light to discriminate short path photons in a multiply scattering medium," Appl. Opt. 31, 6535-6546 (1992).

69. S. P. Morgan, M. P. Khong, and M. G. Somekh, "Effects of polarization state and scatterer concentration on optical imaging through scattering media," Appl. Opt. 36, 1560-1565 (1997).

70. S. P. Schilders, X. S. Gan, and M. Gu, "Resolution improvement in microscopic imaging through turbid media based on differential polarization gating," Appl. Opt. 37, 4300-4302 (1998).

71. P. Shukla, R. Sumathi, S. Gupta, and A. Pradhan, "Influence of size parameter and refractive index of the scatterer on polarization-gated optical imaging through turbid media," J. Opt. Soc. Am. A, 24, 17041713 (2007).

72. R. R. Anderson, "Polarized light examination and photography of the skin," Arch. Dermatol. 127, 1000-1005 (1991).

73. S. L. Jacques and K. Lee, "Polarized video imaging of skin," Proc. SPIE 3245, 356-362 (1998).
74. S. L. Jacques, J. R. Roman, and K. Lee, "Imaging superficial tissues with polarized light," Lasers Surg. Med. 26, 119-129 (2000).

75. S. P. Morgan and I. M. Stockford, "Surface-reflection elimination in polarization imaging of superficial tissue," Opt. Lett. 28, 114-116 (2003).

76. S. G. Demos, H. B. Radousky, and R. R. Alfano, "Deep subsurface imaging in tissues using spectral and polarization filtering," Opt. Express $7,23-28(2000)$

77. W. Groner, J. W. Winkelman, A. G. Harris, C. Ince, G. J. Bouma K. Messmer, and R. G. Nadeau, "Orthogonal polarization spectral imaging: A new method for study of the microcirculation," Nat Med. 5, 1209-1212 (1999).

78. V. Cerny, Z. Turek, and R. Parizkova, "Orthogonal polarization spectral imaging," Physiol. Res. 56, 141-147 (2007).

79. V. Gopal, S. Majumder, H. Ramachandran, and A. K. Sood, "Imaging in turbid media using quasi-ballistic photons," Opt. Сотmun. 170, 331-345 (1999).

80. P. Shukla and A. Pradhan, "Mueller decomposition images for cervical tissue: Potential for discriminating normal and dysplastic states," Opt Express 17, 1600-1609 (2009).

81. N. Ramanujam, "Fluorescence spectroscopy of neoplastic and nonneoplastic tissues," Neoplasia 2, 1-29 (2000).

82. R. Richards Kortum and E. Servick-Muraca, "Quantitative optical spectroscopy for tissue diagnosis," Ann. Rev. Phys. Chem. 47, 556606 (1996).

83. V. Backman, M. B. Wallace, L. T. Perelman, J. T. Arendt, R. Gurjar, M. G. Müller, Q. Zhang, G. Zonios, E. Kline, T. McGillican, S. Shapshay, T. Valdez, K. Badizadegan, J. M. Crawford, M. Fitzmaurice, S. Kabani, H. S. Levin, M. Seiler, R. R. Dasari, I. Itzkan, J. Van Dam, and M. S. Feld, "Detection of preinvasive cancer cells in situ," Nature (London) 406, 35-36 (2000).

84. M. Hunter, V. Backman, G. Popescu, M. Kalashnikov, C. W. Boone, A. Wax, V. Gopal, K. Badizadegan, G. D. Stoner, and M. S. Feld, "Tissue self-affinity and polarized light scattering in the born approximation: a new model for precancer detection," Phys. Rev. Lett. 97, 138102 (2006).

85. H. K. Roy, V. Turzhitsky, Y. L. Kim, Y. Liu, M. J. Goldberg, R. E. Brand, N. Hasabou, M. Jameel, and V. Backman, "Spectral slope from the endoscopically-normal mucosa predicts concurrent colonic neoplasia: A pilot ex-vivo clinical study," Dis. Colon Rectum 51, 13811386 (2008).

86. H. K. Roy, Y. Liu, R. Wali, Y. L. Kim, A. K. Kromine, M. J. Goldberg, and V. Backman, "Four-dimensional elastic light-scattering fingerprints as preneoplastic markers in the rat model of colon carcinogenesis," Gastroenterology 126, 1071-1081 (2004).

87. Y. L. Kim, Y. Liu, R. K. Wali, H. K. Roy, M. J. Goldberg, A. K Kromine, K. Chen, and V. Backman, "Simultaneous measurent of angular and spectral properties of light scattering for characterization of tissue microarchitecture and its alteration in early precancer," IEEE J. Sel. Top. Quantum Electron. 9, 243-257 (2003).

88. V. Turzhitsky, A. J. Gomes, Y. L. Kim, Y. Liu, A. Kromine, J. D. Rogers, M. Jameel, H. K. Roy, and V. Backman, "Measuring mucosal blood supply in vivo with a polarization gating probe," Appl. Opt. 47, 6046-6057 (2008).

89. P. Huang, M. Hunter, and I. Georgakoudi, "Confocal light scattering spectroscopic imaging system for in situ tissue characterization," Appl. Opt. 48, 2595-2599 (2009).

90. N. Ghosh, S. K. Majumder, H. S. Patel, and P. K. Gupta, “19. Depth resolved fluorescence measurement in layered turbid medium by polarized fluorescence spectroscopy," Opt. Lett. 30, 162-164 (2005).

91. N. Ghosh, S. K. Majumder, and P. K. Gupta, "Polarized fluorescence spectroscopy of human tissues," Opt. Lett. 27, 2007-2009 (2002).

92. N. C. Biswal, S. Gupta, N. Ghosh, A. Pradhan, "29. Recovery of intrinsic fluorescence from the measured fluorescence in a turbid medium: an experimental approach," Opt. Express 11, 3320-3331 (2003).

93. Z. J. Smith and A. J. Berger, "Surface-sensitive polarized Raman spectroscopy of biological tissue," Opt. Lett. 30, 1363-1365 (2005).

94. P. J. Wu and J. T. Walsh, Jr., "Stokes polarimetry imaging of rat tail tissue in a turbid medium: degree of linear polarization image maps using incident linearly polarized light," J. Biomed. Opt. 11, 014031 (2006). 
95. R. Ossikovski, A. De Martino, and S. Guyot, "Forward and reverse product decompositions of depolarizing Mueller matrices," Opt. Lett. 32, 689-691 (2007).

96. N. Ghosh, M. F. G. Wood, and I. A. Vitkin, "Influence of the order of the constituent basis matrices on the Mueller matrix decompositionderived polarization parameters in complex turbid media such as biological tissues," Opt. Commun. 283, 1200-1208 (2010).

97. R. Ossikovski, "Analysis of depolarizing Mueller matrices through a symmetric decomposition," J. Opt. Soc. Am. A 26, 1109-1118 (2009).

98. R. Ossikovski, M. Anastasiadou, S. Ben Hatit, E. Garcia-Caurel, and A. De Martino, "Depolarizing Mueller matrices: how to decompose them?" Phys. Status Solidi A 205, 720-727 (2008).

99. N. Ghosh, M. F. G. Wood, and I. A. Vitkin, "Polarimetry in turbid, birefringent, optically active media: a Monte Carlo study of Mueller matrix decomposition in the backscattering geometry," J. Appl. Phys. 105, 102023 (2009).

100. M. F. G. Wood, N. Ghosh, E. H. Moriyama, B. C. Wilson, and I. A. Vitkin, "Proof-of-principle demonstration of a Mueller matrix decomposition method for polarized light tissue characterization in vivo," J. Biomed. Opt. 14, 014029 (2009).

101. M. F. G. Wood, D. Côté, and I. A. Vitkin, "Combined optical intensity and polarization methodology for analyte concentration determination in simulated optically clear and turbid biological media," J. Biomed. Opt. 13, 044037 (2008).

102. D. J. Maitland and J. T. Walsh, Jr., "Quantitative measurements of linear birefringence during heating of native collagen," Lasers Surg. Med. 20, 310-318 (1997).

103. B. H. Park, C. Saxer, S. M. Srinivas, J. S. Nelson, and J. F. de Boer, "In vivo burn depth determination by high-speed fiber-based polarization sensitive optical coherence tomography," J. Biomed. Opt. 6, 474-479 (2001).

104. S. Manhas, M. K. Swami, H. S. Patel, A. Uppal, N. Ghosh, and P. K. Gupta, "Polarized diffuse reflectance measurements on cancerous and noncancerous tissues," J. Biophoton. 2, 581-587 (2009).
105. M. Anastasiadou, A. De Martino, D. Clement, F. Liège, B. LaudeBoulesteix, N. Quang, J. Dreyfuss, B. Huynh, A. Nazac, L. Schwartz, and H. Cohen, "Polarimetric imaging for the diagnosis of cervical cancer," Phys. Status Solidi C 5, 1423-1426 (2008).

106. D. Orlic, J. Kajstura, S. Chimenti, I. Jakoniuk, S. M. Anderson, B. Li, J. Pickel, R. McKay, B. Nadal-Ginard, D. M. Bodine, A. Leri, and P. Anversa, "Bone marrow cells regenerate infarcted myocardium," Nature 410, 701-705 (2001).

107. M. F. G. Wood, N. Ghosh, M. A. Wallenburg, S.-H. Li, R. D. Weisel, B. C. Wilson, R.-K. Li, and I. A. Vitkin, "Polarization birefringence measurements for characterizing the myocardium, including healthy, infracted, and stem cell treated regenerating cardiac tissues," J. Biomed. Opt. 15, 047009 (2010).

108. M. A. Wallenburg, M. F. G. Wood, N. Ghosh and I. A. Vitkin, "Polarimetry-based method to extract geometry-independent metrics of tissue anisotropy," Opt. Lett. 35, 2570-2572 (2010).

109. M. A. Wallenburg, M. Pop, M. F. G. Wood, N. Ghosh, G. A. Wright, and I. A. Vitkin, "Comparison of optical polarimetry and diffusion tensor MR imaging for assessing myocardial anisotropy," J. Innov. Opt. Health Sci. 3, 109-121 (2010).

110. Jungrae Chung, Woonggyu Jung, Marie J. Hammer-Wilson, Petra Wilder-Smith, and Zhongping Chen, "Use of polar decomposition for the diagnosis of oral precancer," Appl. Opt. 46, 3038-30 44 (2007).

111. A. Pierangelo, A. Benali, M.-R. Antonelli, T. Novikova, P. Validire, B. Gayet, and A. De Martino, "Ex-vivo characterization of human colon cancer by Mueller polarimetric imaging," Opt. Express 19, 1582-1593 (2011).

112. X. Li and G. Yao, "Mueller matrix decomposition of diffuse reflectance imaging in skeletal muscle," Appl. Opt. 48, 2625-2631 (2009).

113. H. He, Z. Nan, L. Ran, Y. Tianliang, L. Wei, H. Yonghong, and M. Hui, "Application of sphere-cylinder scattering model to skeletal muscle," Opt. Express 18, 15104-15112 (2010).

114. J. F. de Boer and T. E. Milner, "Review of polarization sensitive optical coherence tomography and Stokes vector determination," J. Biomed. Opt. 7, 359-371 (2002). 